\title{
Identification and characterisation of a phospholipid scramblase in the malaria parasite Plasmodium falciparum
}

\author{
Silvia Haase $^{\mathrm{a}, *}$, Melanie Condron ${ }^{\mathrm{b}}$, David Miller ${ }^{\mathrm{c}, 1}$, Dounia Cherkaoui ${ }^{\mathrm{a}, 2}$, Sarah Jordan ${ }^{\mathrm{a}}$, \\ Jacqueline M Gulbis ${ }^{c, d}$, Jake Baum ${ }^{\mathrm{a}, *}$ \\ ${ }^{a}$ Department of Life Sciences, Imperial College London, Sir Alexander Fleming Building, Exhibition Road, South Kensington, London, UK \\ ${ }^{\mathrm{b}}$ Division of Infection and Immunity, The Walter and Eliza Hall Institute of Medical Research, Melbourne, Victoria, Australia \\ ${ }^{\mathrm{c}}$ Division of Structural Biology, The Walter and Eliza Hall Institute of Medical Research, Melbourne, Victoria, Australia \\ ${ }^{\mathrm{d}}$ Department of Medical Biology, The University of Melbourne, Melbourne, Victoria, Australia
}

\section{A R T I C L E I N F O}

\section{Keywords:}

Malaria

Plasmodium falciparum

Invasion

Phospholipid scramblase

Liposomes

Gametocytes

\begin{abstract}
A B S T R A C T
Recent studies highlight the emerging role of lipids as important messengers in malaria parasite biology. In an attempt to identify interacting proteins and regulators of these dynamic and versatile molecules, we hypothesised the involvement of phospholipid translocases and their substrates in the infection of the host erythrocyte by the malaria parasite Plasmodium spp. Here, using a data base searching approach of the Plasmodium Genomics Resources (www.plasmodb.org), we have identified a putative phospholipid (PL) scramblase in $P$. falciparum (PfPLSCR) that is conserved across the genus and in closely related unicellular algae. By reconstituting recombinant PfPLSCR into liposomes, we demonstrate metal ion dependent PL translocase activity and substrate preference, confirming PfPLSCR as a bona fide scramblase. We show that PfPLSCR is expressed during asexual and sexual parasite development, localising to different membranous compartments of the parasite throughout the intra-erythrocytic life cycle. Two different gene knockout approaches, however, suggest that PfPLSCR is not essential for erythrocyte invasion and asexual parasite development, pointing towards a possible role in other stages of the parasite life cycle.
\end{abstract}

\section{Introduction}

Infection of the host erythrocyte by the malaria parasite Plasmodium spp. is a crucial event in the pathobiology of this pathogen. The process of erythrocyte invasion involves a highly orchestrated cascade of protein-protein interactions between the parasite and the host cell. Once inside, the asexual, intra-erythrocytic proliferation of Plasmodium spp. is accompanied by a high demand of lipids. These lipids are required to build membranes for daughter cell and organelle neogenesis, promote the expansion of the parasitophorous vacuole (PV) in which the parasite resides and develops, and to generate a parasite-derived exomembrane system involved in parasite virulence and nutrient uptake (reviewed in $[1,2])$. Accordingly, the progression of blood-stage parasites from ring to schizont stages is marked by increased metabolic activity and stage-specific changes in the lipidomic profiles within the infected erythrocyte [3]. Individual phospholipids have also come into focus by playing fundamental roles in signalling. Phosphoinositides have been implicated in merozoite development [4], haemoglobin digestion [5], microneme secretion [6] and apicoplast biogenesis in Plasmodium spp. and its apicomplexan relative Toxoplasma gondii $[7,8]$. Another important lipid messenger, phosphatidic acid, has also been linked to microneme exocytosis [9] and host cell egress in Toxoplasma gondii [10]. Both, the apicoplast (a non-photosynthetic, relict plastid shared by most members of the phylum Apicomplexa) and the micronemes (specialised organelles containing adhesins involved in erythrocyte invasion) are essential to parasite survival. Lipid rafts have also been implicated in facilitating and regulating host-pathogen interactions in different protozoan parasites, including Plasmodium spp. and the intestinal pathogens Giardia intestinales and Entamoeba histolytica (reviewed in [11]). Investigations into the roles played by lipids and their interacting proteins and regulators are therefore increasingly seen as an attractive avenue to explore and to identify novel antimalarial and antiparasitic drug targets.

\footnotetext{
* Corresponding authors.

E-mail addresses: silvia.haase@crick.ac.uk (S. Haase), jake.baum@imperial.ac.uk (J. Baum).

1 Present address: Advanced Hackspace, Imperial College London, White City, London, UK.

2 Present address: The London Centre for Nanotechnology, Bloomsbury, London, UK.
} 
Prompted by the growing evidence and importance of lipid function in malaria parasite and apicomplexan biology, as well as in bacterial and viral infections (reviewed in [12]), we sought to investigate the role of putative phospholipid (PL) translocases and their substrates in the infection of the host erythrocyte by the most virulent human malaria parasite $P$. falciparum, which causes an estimated $\sim 400,000$ fatalities and $\sim 200$ million cases per year worldwide (WHO report 2019, www. who.int). An initial search for candidates in the Plasmodium Genomics Resource (www.plasmodb.org) revealed a predicted and so far uncharacterised PL scramblase which fulfilled all characteristics of our search rationale: (i) putative PL translocase activity, (ii) maximal expression in the late blood stages and (iii) predicted localisation to the plasma membrane in the invasive stage, the merozoite.

Phospholipids are asymmetrically distributed in the plasma membrane. While phosphatidylcholine (PC) and sphingomyelin (SM) dominate the outer leaflet of the lipid bilayer, phosphatidylserine (PS), phosphatidylethanolamine (PE) and phosphatidylinositol (PI) characterise the inner leaflet (reviewed in [13]). Phospholipid asymmetry is maintained by PL translocases, such as flippases and floppases, which function as ATP-dependent unidirectional transporters that move PLs from the outer to the cytosolic face or from the inner to the exoplasmic face, respectively (reviewed in [14]). In contrast, PL scramblases, including members of the PLSCR and TMEM16 families, are activated by an increase of intracellular $\mathrm{Ca}^{2+}$ in response to cell stress or extracellular signals such as apoptotic stimuli and mediate the bidirectional movement of PLs across the bilayer. Consequently, PS and other lipids normally confined to the inner leaflet become exposed at the cell surface and aid in the process of blood coagulation and clearance of dead or injured cells (reviewed in [15]). $\mathrm{Ca}^{2+}$ signalling plays an important role in Plasmodium spp. biology being involved in different processes such as host erythrocyte invasion and egress (reviewed in [16]). Phosphatidylserine has also been found to be exposed in the HIV-1 envelope, thereby mediating virus attachment, entry and replication (reviewed in [17]). Combined, these latter observations give further impetus to investigate the newly identified and likely $\mathrm{Ca}^{2+}$-dependent PL scramblase in $P$. falciparum, hereafter referred to as PfPLSCR, and a possible role in erythrocyte invasion and asexual parasite development.

The human genome encodes four members of the PLSCR family (hPLSCR1 -4); with the exception of hPLSCR2, these proteins have been reported to be expressed in various tissues [18]. The best studied member to date is hPLSCR1; initially identified in and purified from erythrocytes $[19,20]$, the protein is characterised by several functional domains: an N-terminal proline-rich domain containing multiple PXXP and PPXY motifs suggested to mediate protein-protein interactions [18]; a DNA binding motif spanning $\mathrm{Met}^{86}$ to Glu ${ }^{118}$ which has been implicated in binding the promoter region of the inositol 1,4,5-triphosphate receptor type I [21]; a palmitoylation site ${ }^{184} \mathrm{CCCPCC}^{189}$ involved in trafficking to the plasma membrane [22]; a non-classical nuclear leader sequence (NLS) ${ }^{257}$ GKISKHWTGI ${ }^{266}$ shown to bind importin $\alpha$ [23] precedes an EF-hand like $\mathrm{Ca}^{2+}$ binding motif ${ }^{273} \mathrm{D}$ [A/S]DNFGIQFPLD ${ }^{284}$ which is directly followed by a C-terminal $\alpha$-helical stretch suggested to insert hPLSCR1 into artificial bilayers [20,24,25]. The integral membrane protein is expressed in blood cells and a wide range of tissues and cancer cell lines $[19,20,26]$ and is capable of translocating PLs in reconstituted membrane systems [19,24,27-29]. While its actual cellular role in PL scrambling remains a matter of debate, hPLSCR1 has been implicated in a range of different activities related to apoptosis, transcriptional regulation, autoimmunity, antiviral defence and the development of cancer and lipid-related diseases (reviewed in [15]).

In this study, we biochemically characterised recombinant PfPLSCR and applied a gene tagging approach to investigate the expression and localisation of endogenously expressed PfPLSCR-HA in transgenic $P$. falciparum parasites. In addition, we have utilised two different gene knockout strategies to analyse the loss of function phenotype in mutant and PfPLSCR deficient parasite lines. Though the function of the protein remains elusive, our findings support the biochemical and cellular observations made for the human ortholog hPLSCR1 and may suggest a still to be determined regulatory role in parasite sexual development.

\section{Materials and methods}

\subsection{Recombinant protein expression and inclusion body solubilisation}

Codon-optimised full-length Pfplscr was synthesised as a gBlock ${ }^{\circledR}$ (Integrated DNA Technologies) and cloned into the BamHI/XhoI site of the pPROEX HTa vector (Invitrogen), allowing the expression of recombinant PfPLSCR with an N-terminal hexa-histidine affinity tag (His:: PfPLSCR) in E. coli BL21 (DE3) cells. Protein expression was induced with $1 \mathrm{mM}$ IPTG for $14-16 \mathrm{~h}$ at $16{ }^{\circ} \mathrm{C}$. Bacterial cells were pelleted and lysed in lysis buffer [250 mM NaCl, $0.5 \mathrm{mM} \mathrm{CaCl}_{2}, 1 \mathrm{mM} \mathrm{MgCl}_{2}, 50 \mathrm{mM}$ HEPES pH 7.5] using a high-pressure cell disruptor (Constant Systems). Insoluble material was then centrifuged at $30,000 \mathrm{~g}\left(30 \mathrm{~min}, 4^{\circ} \mathrm{C}\right)$ and washed twice with wash buffer $\left[0.5 \mathrm{M} \mathrm{NaCl}, 0.5 \mathrm{mM} \mathrm{CaCl}_{2}, 1 \mathrm{mM}\right.$ $\mathrm{MgCl}_{2}, 50 \mathrm{mM}$ HEPES $\mathrm{pH}$ 7.5] by sonication and centrifugation as described above. Inclusion bodies were solubilised in solubilisation buffer [250 mM NaCl, $0.5 \mathrm{mM} \mathrm{CaCl}_{2}, 1 \mathrm{mM}$ TCEP, $1 \%$ SDS, $25 \mathrm{mM}$ HEPES pH 7.5] under gentle agitation over night at $25^{\circ} \mathrm{C}$ and the soluble fraction collected by centrifugation at $30,000 \mathrm{~g}\left(30 \mathrm{~min}, 25^{\circ} \mathrm{C}\right)$. All buffers contained cOmplete ${ }^{\mathrm{TM}}$ EDTA-free protease inhibitors (Roche).

\subsection{Refolding and purification of His::PfPLSCR}

Recovered His::PfPLSCR protein was refolded by rapid dilution into IMAC buffer A [250 mM NaCl, $0.5 \mathrm{mM} \mathrm{CaCl}_{2}, 1 \mathrm{mM}$ TCEP, $0.1 \%$ Foscholine-12 (n-Dodecyl-phosphocholine, Anatrace), 25 mM HEPES pH 7.5], resulting in a final SDS concentration of $0.1 \%$. Refolded protein was filtered through a $0.45 \mu \mathrm{m}$ filter prior to immobilised metal affinity chromatography (IMAC) using a HisTRAP ${ }^{\mathrm{TM}}$ High Performance column (GE Healthcare), which was equilibrated with IMAC buffer A.

The loaded protein column was washed with several column volumes of wash buffer [IMAC buffer A $+0.4 \%$ SDS] and IMAC buffer A. The protein was eluted in a linear imidazole gradient using IMAC buffer B [IMAC buffer A $+1 \mathrm{M}$ imidazole]. Fractions containing the desired protein were concentrated (Amicon Ultracel $100 \mathrm{~K}$, Millipore) and further purified by size-exclusion chromatography (SEC) using a Superdex S200 10/300 G L column (GE Healthcare) in SEC buffer [250 $\mathrm{mM} \mathrm{NaCl}, 0.5 \mathrm{mM} \mathrm{CaCl}_{2}, 1 \mathrm{mM}$ TCEP, $0.1 \%$ FC-12, $25 \mathrm{mM}$ HEPES pH 7.5]. All buffers contained cOmplete ${ }^{\mathrm{TM}}$ EDTA-free protease inhibitors (Roche). Purity of the protein was assessed by SDS-PAGE using NuPAGETM Novex ${ }^{\circledR}$ Bis-Tris gels and blue native PAGE was performed by using the NativePAGE ${ }^{\mathrm{TM}}$ Novex ${ }^{\circledR}$ Bis-Tris Gel System and G250 additive (Life Technologies).

\subsection{Intrinsic tryptophan fluorescence}

Intrinsic tryptophan fluorescence of recombinant protein was measured using a Cary Eclipse Spectrophotometer (Agilent) and Jasco FP-8500 Spectrofluorometer, respectively. Cuvettes were washed with Helmanex ${ }^{\mathrm{TM}} \mathrm{III}$ and rinsed with ion-free water before each use. Fresh stocks of $\mathrm{CaCl}_{2}$ and $\mathrm{MgCl}_{2}$ were prepared using ion-free water for metal ion titration and measurements in reconstitution buffer $[100 \mathrm{mM} \mathrm{NaCl}$, 10 mM HEPES, pH 7.5] [30] containing either 2 mM EGTA (for cation free conditions) or $2 \mathrm{mM}$ EGTA and the desired metal ion concentration as calculated by using the Maxchelator programme [https://somapp.uc dmc.ucdavis.edu].

Five $\mu \mathrm{g}$ of purified recombinant protein were used per emission scan (in either duplicate or triplicate measurements per condition and experiment). Intrinsic fluorescence emission spectra were recorded from 310 to $470 \mathrm{~nm}$ at $25{ }^{\circ} \mathrm{C}$ and an excitation wavelength of $295 \mathrm{~nm}$. Emission and excitation bandwidths were set to $5 \mathrm{~nm}$ and $10 \mathrm{~nm}$, respectively. Equilibrium dissociation constants were determined by using the nonlinear regression analysis tool and one-site total binding 
equation (GraphPad Prism) as described in [28].

\subsection{Reconstitution of recombinant His::PfPLSCR into liposomes}

The following lipids were obtained from Avanti Polar Lipids to prepare liposomes: 1-palmitoyl-2-oleoyl-sn-glycero-3-phosphocholine (POPC); 1-palmitoyl-2-oleoyl-sn-glycero-3-phosphoethanol-amine (POPE); 1,2-di-oleoyl-sn-glycero-3-[(N-(5-amino-1-carboxypentyl) iminodiacetic acid) succinyl] nickel salt (18:1 DGS-NTA (Ni)); 1,2-dioleoylsn-glycero-3-phospho-L-serine-N-(7-nitro-2-1,3- benzoxadiazol-4-yl) (18:1 NBD-PS) and 1,2-dioleoyl-sn-glycero-3-phosphoethanolamine-N(7-nitro-2-1,3-benzoxadiazol-4-yl) (18:1 NBD-PE).

To prepare liposomes, dried lipid films of either PC only or PC/PE (70:30 \% w/w), supplemented with $0.1 \%$ DGS-NTA (Ni) and either 0.25 $\%$ NBD-PE $(\mathrm{w} / \mathrm{w})$ or $0.25 \%$ NBD-PS $(\mathrm{w} / \mathrm{w})$, respectively, were hydrated at $3 \mathrm{mg} / \mathrm{mL}$ total lipid in reconstitution buffer and incubated for $1 \mathrm{~h}$ at $37^{\circ} \mathrm{C}$ under agitation. The emulsion was then sonicated in a sonicator bath for $1 \mathrm{~min}$ and subjected to five freeze-thaw cycles. Uniformly sized ULVs (unilamellar vesicles) were obtained using a lipid extruder mounted with a $100 \mathrm{~nm}$ membrane (Avanti Polar Lipids).

To prepare proteoliposomes, liposomes were destabilised with nDodecyl- $\beta$-D-Maltopyranoside (DDM, Anatrace) at 1:8 $\mathrm{M}$ ratio (detergent to lipid) for $15 \mathrm{~min}$ at $25{ }^{\circ} \mathrm{C}$ prior to the addition of purified His:: PfPLSCR (5 $\mu \mathrm{g}$ protein per $\mathrm{mg}$ of lipid, adapted from [31]). Aliquots of detergent treated liposomes were taken aside, matching volumes of SEC buffer were added instead of protein and liposome only controls were treated as proteoliposomes which were formed under gentle agitation and incubated at $25{ }^{\circ} \mathrm{C}$ for $24 \mathrm{~h}$. Detergent was removed by adding excess amounts $(\sim 100$-fold $)$ of reconstitution buffer and vesicles were collected by ultracentrifugation at $150,000 \mathrm{~g}\left(1 \mathrm{~h}, 25{ }^{\circ} \mathrm{C}\right)$ and resuspended in reconstitution buffer containing $2 \mathrm{mM}$ EGTA and at a lipid concentration of $\sim 10 \mathrm{mg} / \mathrm{mL}$.

\subsection{Scramblase assay}

Based on established methods to characterise PL scramblases [24, 27-29,31,32], phospholipid translocase activity of recombinant His:: PfPLSCR was measured using a Cary Eclipse Spectrophotometer (Agilent) and Jasco FP-8500 Spectrofluorometer, respectively. Stirring cuvettes and bars were rinsed with Helmanex ${ }^{\mathrm{TM}}$ III and ion-free water before each use. Five $\mu \mathrm{l}$ of proteoliposome (or liposome only) suspension were diluted in $1 \mathrm{~mL}$ of reconstitution buffer containing either $2 \mathrm{mM}$ EGTA or $2 \mathrm{mM}$ EGTA and the desired metal ion concentration and stirred at $25{ }^{\circ} \mathrm{C}$. Emission spectra from 485 to $600 \mathrm{~nm}$ were recorded at $25{ }^{\circ} \mathrm{C}$ and an excitation wavelength of $470 \mathrm{~nm}$ to determine the exact emission maxima of the NBD moieties (533 nm for NBD-PE and $546 \mathrm{~nm}$ for NBD-PS). Initial fluorescence was recorded for $100 \mathrm{~s}$ prior to the addition of freshly prepared sodium dithionite (in unbuffered $0.5 \mathrm{M}$ Tris) to a final concentration of $30 \mathrm{mM}$. Fluorescence decay was recorded for the next $400 \mathrm{~s}$ and maximal activity was determined by dividing $\mathrm{F}=$ fluorescence at Time $=500 \mathrm{~s}$ by $\mathrm{F}_{\max }=$ fluorescence prior to addition of 30 $\mathrm{mM}$ sodium dithionite. Traces were fitted using the 'Plateau followed by one phase decay' equation in GraphPad Prism.

\subsection{Transfection constructs}

We used a recently published conditional gene deletion vector [33]

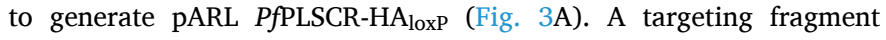
composed of the full length Pfplscr coding sequence followed by a triple hemagglutinin tag (HA), as well as two synthetic loxPint modules [34] flanking the recodonised sequence encoding the C-terminal protein region Lys ${ }^{217}$-Lys $^{275}$ was synthesised (Geneart ${ }^{\circledR}$ ) and cloned into PARL FIKK10.1:loxPint: HA (a kind gift from the Treeck lab) by removing the fikk10.1 gene via BglII/PstI.

Full Pfplscr gene disruption was attempted using CRISPR-Cas9 and by replacing the gene locus with a superfolder green fluorescent protein
(sfGFP) expression cassette (Fig. 6A). To generate the donor plasmid pKW003_1022700, primers $10227005 \mathrm{~F}$ and $\mathrm{R}$ were used to amplify the 572 bp 5' homology flank and primer pair $10227003 \mathrm{~F}$ and $\mathrm{R}$ was used to amplify the 673 bp 3' homology flank (KOD Hot Start DNA Polymerase, Merck Millipore) which were cloned on either side of the sfGFP expression cassette in pkiwi003 [35] using appropriate restriction sites (Table S1). The pDC2-cam-coCas9-U6.2-hDHFR vector [36] was used to clone in either of the guide RNAs 951969, 952497 and 952530, which were identified by using the web tool CHOPCHOPv2 (http://chopchop. cbu.uib.no [37]). Therefore, two complimentary oligos (of each guide) with 5' TATT (oligo 1) and 5' AAAC (oligo 2) overhangs were annealed for ligation into the BbsI digested and dephosphorylated pDC2-cam-coCas9-U6.2-hDHFR vector (a kind gift from the Lee lab).

\subsection{Parasite culture and transfection}

P. falciparum asexual parasites were cultured in RPMI 1640 medium containing $0.5 \% \mathrm{w} / \mathrm{v}$ AlbuMax II ${ }^{\circledR}$ (Gibco) and at $4 \%$ haematocrit using human erythrocytes (blood group $0^{+}$) according to standard procedures [38]. Ring-stage parasites were synchronised using sorbitol treatment. Tightly sychronised DiCre-expressing B11 parasites [39] were transfected with $100 \mu \mathrm{g}$ of purified pARL PfPLSCR-HA loxP $_{\text {plasmid and }}$ initially selected with $2.5 \mathrm{nM}$ WR99210 (Jacobus Pharmaceuticals) to obtain transgenic parasites harbouring the episomal plasmid. Integration into the genomic locus was achieved by G418 treatment (400 $\mu \mathrm{g} / \mathrm{mL}$ ) to select for integrants as described in [40].

DiCre-mediated recombination was induced by treating synchronised ring-stage parasites with $100 \mathrm{nM}$ rapamycin (Sigma) in $1 \%(\mathrm{v} / \mathrm{v})$ DMSO for 14-16 h to achieve complete excision. In parallel, parasites were mock-treated with $1 \%(\mathrm{v} / \mathrm{v})$ DMSO as a negative control. Treated parasites were transferred into $96-$ well plates at $0.1 \%$ parasitemia (in triplicate) containing fresh red blood cells at $0.1 \%$ haematocrit and were allowed to proceed to the next ring-stage cycle for flow cytometry. Samples for gDNA extraction and PCR amplification, Western blot and immunofluorescence analysis were taken towards the end of the treatment cycle or subsequent cycles.

Ring-stage 3D7 parasites were co-transfected with different combinations of either guide RNA (and Cas9) containing plasmid 951969, 953497 and 953530, as well as with pKW003_1022700 donor plasmid (50 $\mu$ g each). Drug selection with WR99210 was carried out for 6 days post transfection to ensure sufficient expression of the Cas9 nuclease. Rapid loss of the donor plasmid was assumed in the absence of any selectable marker and sfGFP positive parasites would therefore only be obtained upon successful replacement of the Pfplscr gene with the sfGFP expression cassette.

\subsection{P. falciparum gametocyte culture}

Asexual parasite cultures were adapted to gametocyte culture media [RPMI 1640 medium containing $5 \%$ pooled human serum, $5 \%$ AlbuMax II $\AA$, 4\% sodium bicarbonate and $3.5 \%$ Hypoxanthine-Thymidine supplement (Sigma-Aldrich)] prior to the induction of gametocytes. Gametocyte cultures were seeded from synchronised asexual cultures at high ring-stage parasitemia $(\sim 5-10 \%)$ and $4 \%$ haematocrit and were maintained at $37{ }^{\circ} \mathrm{C}$ under $3 \% \mathrm{O}_{2} / 5 \% \mathrm{CO}_{2} / 93 \% \mathrm{~N}_{2}$ gas (BOC, UK). Spent culture media was replaced daily for 14 days and cultures were treated with heparin $(20 \mathrm{U} / \mathrm{mL})$ on day 4 post induction for four days to kill uncommitted asexual parasites.

\subsection{Solubility analysis}

Stage-specific infected RBCs were lysed with $0.02 \%$ saponin/PBS. Released parasite material was washed three times with PBS (containing protease inhibitors) prior to hypotonic lysis with $5 \mathrm{mM} \mathrm{TrisCl}(\mathrm{pH} \mathrm{8.0)}$ for $1 \mathrm{~h}$ on ice. The soluble protein fraction was collected by ultracentrifugation at $100,000 \mathrm{~g}$ for $30 \mathrm{~min}$ at $4{ }^{\circ} \mathrm{C}$. The pellet was resuspended in 
$100 \mathrm{mM} \mathrm{Na}_{2} \mathrm{CO}_{3}$ ( $\mathrm{pH}$ 11.2) to extract peripheral membrane proteins for $1 \mathrm{~h}$ on ice. The carbonate-soluble fraction was collected as above and the carbonate-insoluble pellet was needle-passed with $1 \%$ Triton X-100/ PBS and incubated at $37{ }^{\circ} \mathrm{C}$ for $30 \mathrm{~min}$ to extract integral membrane proteins. Triton X-100 soluble and insoluble fractions were separated as above and equal amounts of all fractions were analysed by immunoblotting.

\subsection{Western blot analysis}

For immunoblots, infected RBCs were lysed and washed as above. Parasite material was resuspended in 1x SDS sample buffer. Extracted proteins were separated on NuPAGETM ${ }^{\mathrm{TM}}{ }^{\circledR}{ }^{\circledR} 4-12 \%$ Bis-Tris protein gels in MES buffer (Life Technologies) and transferred onto nitrocellulose membranes using the iBlot ${ }^{\circledR}$ system (Life Technologies). Rabbit anti-HA (clone C29F4, Cell Signaling) was diluted 1:4,000; mouse antiHA (clone 12CA5, Roche), mouse anti-GFP (clones 7.1 and 13.1, Roche), rabbit anti-ADF1 [41], rabbit anti-F-Actin [42] and mouse anti-Pfs16 (a kind gift from Professor Robert Sauerwein) were diluted 1:1,000 in $5 \%$ skim milk/PBS (w/vol). Horseradish peroxidase-conjugated goat anti-rabbit and goat anti-mouse antibodies were used as secondary antibodies (Jackson IR) and diluted 1:10,000 in $5 \%$ skim milk/PBS.

\subsection{Immunofluorescence analysis and imaging}

Infected RBCs were fixed with $4 \%$ paraformaldehyde and $0.0075 \%$ glutaraldehyde in PBS for 30 min, permeabilised with $0.1 \%$ Triton X100/PBS for $10 \mathrm{~min}$, and blocked in $3 \% \mathrm{BSA}$ PBS prior to immunolabelling with the following primary antibodies diluted in $3 \% \mathrm{BSA} /$ PBS: Rabbit anti-HA (1:1,000); mouse anti-HA (1:500), mouse anti-GFP (1:100), rabbit anti-MSP1 (1:500, [43]), rabbit anti-SPP (1:100 [44],), rabbit anti-EXP1 (1:100), mouse anti-Pfs16 (1:1000) and rabbit anti-GAP45 (1:500 [45],). Appropriate Alexa Fluor ${ }^{\circledR}$ conjugated secondary antibodies (Life Technologies) and DAPI (4',6-diamidino-2-phenylindole) were diluted 1:4,000 in $3 \%$ BSA/PBS. Images were captured using a Nikon Ti Microscope and OrcaFlash4.0 digital camera and were processed with Fiji [46]. Z-stacks were deconvolved using the EpiDEMIC plugin with 80 iterations in Icy [47].

\subsection{Flow cytometry}

Tightly synchronised ring-stage parasites were diluted to $0.1 \%$ parasitemia (in triplicate) for growth analysis and transferred into 96well plates containing fresh red blood cells at $0.1 \%$ haematocrit. Parasites were allowed to proceed to the next ring-stage cycle and were incubated with SYBR Green $\left(1: 10,000\right.$, Sigma) for $10 \mathrm{~min}$ at $37{ }^{\circ} \mathrm{C}$ in the dark. Stained cells were washed three times with PBS prior to collection by flow cytometry using a BD LSRFortessa ${ }^{\mathrm{TM}}$. A 100,000 cells were counted, FCS-A vs SSC-A was used to gate for red blood cells, FCS-A vs FCS-H for singlets and SYBR-A vs FCS-H was applied to gate for infected red blood cells (Fig. S3). Flow cytometry data was analysed in FlowJo. Biological replicates are indicated in the figures.

\section{Results}

\subsection{P. falciparum encodes a putative PL scramblase}

In order to identify putative PL translocases, we used three search criteria available in the Plasmodium Genomics Resource database (www. plasmodb.org): (i) Function prediction as determined by EC number and GO term, (ii) transcriptomics data and (iii) protein features and properties. This search identified a single exon gene (gene identifier PF3D7_1022700) encoding for a putative PL scramblase of approx. 33 $\mathrm{kDa}$, hereafter referred to as PfPLSCR (Fig. 1A), which is predicted to be highly upregulated in late schizont stages ( $40-48 \mathrm{~h}$ post invasion) and to associate with the plasma membrane.
A protein sequence alignment of PfPLSCR with hPLSCR1 revealed moderate sequence conservation between the two proteins sharing only $17 \%$ sequence identity and $31 \%$ sequence similarity (www.bioinfor matics.org/sms2/identsim.html [48]). However, all characteristic domains of hPLSCR1 appear to be partially or fully conserved in the parasite homolog (Fig. 1B). The N-terminus of PfPLSCR is shorter but contains several proline residues with a possible PXXP motif. A putative, semi-conserved DNA binding region $\mathrm{Ser}^{5 \circ}$ to $\mathrm{Glu}^{65}$ precedes two predicted palmitoylation sites ${ }^{172} \mathrm{CC}^{173}$ and ${ }^{194} \mathrm{CC}^{195}$ (http://csspalm.bioc uckoo.org [49]) and a predicted bipartite NLS which starts at $\mathrm{Arg}^{228}$ (http://nls-mapper.iab.keio.ac.jp [50]) and partially overlaps with the putative $\mathrm{Ca}^{2+}$ binding site. Several residues of the $\mathrm{Ca}^{2+}$ binding region ${ }^{240}$ DIDNYTLDFEQV ${ }^{251}$, most notably in positions $1\left(\mathrm{Asp}^{240}\right), 3\left(\mathrm{Asp}^{242}\right)$ and $9\left(\mathrm{Phe}^{248}\right)$, as well as its positioning adjacent to the C-terminal transmembrane helix spanning Pro ${ }^{254}$ to $\mathrm{Arg}^{274}$ (http://topcons.cbr.su. se [51]) are conserved in the putative PL scramblase of $P$. falciparum, indicating a functional preservation of these domains in the parasite protein.

\subsection{PfPLSCR is conserved within Plasmodium spp. but not in other Apicomplexa}

Orthologs of hPLSCR1 are present in various organisms ranging from fungi and plants to animals (reviewed in [52]). A BLAST analysis against the Eukaryotic Pathogen database (www.eupathdb.org) revealed putative PLSCR proteins across the genus Plasmodium. Protein sequence identities to PfPLSCR range from approx. $68 \%$ for the orthologs of the rodent malaria parasites $P$. berghei and $P$. yoelii to $97 \%$ for the putative PLSCR of $P$. gaboni, a chimpanzee infecting member of the $P$. falciparum lineage (Fig. 1C). While the N-termini of the Plasmodium spp. PLSCR proteins displayed some variation in length and in the abundance of proline residues, the functional domains of the C-terminal portion appeared to be highly conserved as previously described for other PLSCR orthologs (reviewed in [52]). No putative PLSCR proteins could be identified in the remaining Apicomplexa or unicellular pathogens, including members of the phyla Amoebozoa, Diplomonadida, Kinetoplastida, Oomycetes and Trichomonanida, pointing towards a specific role of the PL scramblase in malaria parasite biology.

The search, however, did identify orthologs in the photosynthetic algae Chromera velia and Vitrella brassicaformis, sharing 24-28\% sequence identity and $37-42 \%$ sequence similarity with the putative PL scramblase of $P$. falciparum (Fig. S1). The algae represent the closest known relative of apicomplexan parasites and are classified as Chromerida, a marine phylum belonging to an ancient group of unicellular eukaryotes, the Alveolata, which are characterised by flattened, membranous sacs (alveoli) just underneath the plasma membrane [53-55].

\subsection{Expression and purification of recombinant PfPLSCR}

To biochemically characterise the putative PfPLSCR and validate its potential PL translocase activity, we recombinantly expressed the fulllength protein with a N-terminal hexa-histidine affinity tag (His:: $P f$ PLSCR) in E. coli BL21(DE3). Several attempts to obtain biologically active His::PfPLSCR using previously described methods for recombinant hPLSCR1 failed $[27,30]$ and required further optimisation to recover functional His::PfPLSCR from inclusion bodies (Fig. 2A). The solubilised protein was refolded by rapid dilution prior to immobilised metal affinity chromatography and gel filtration. Optimal protein stability was achieved by using the zwitterionic surfactant Fos-Choline-12 as determined in a comprehensive detergent screen.

A recent study provided first evidence for $\mathrm{Ca}^{2+}$-induced oligomerisation of hPLSCR1 which in turn activates PL scrambling [28]. Hence, all steps of inclusion body solubilisation, protein refolding and purification of His::PfPLSCR were carried out in the presence of $0.5 \mathrm{mM} \mathrm{Ca}^{2+}$ and Fos-Choline-12. Association of detergent micelles with membrane proteins is known to obscure the elution behavior in size-exclusion 
A

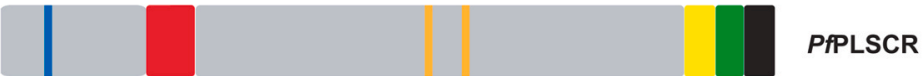

B
PEPLSCR
MEEKNIHMQ-----------------------------PNI-----------------N hPLSCR1
MDKQNSQMNA
*.
.
13
YSYRNPNMYNMNYHNP IVPP PQQQMQLFVNDWKS ILSPMQTC-------------KIKQQ
PEPLSCR
YSYRNPNMYNMNYHNPIVPPPQQQMQLFVNDWKS I LSPMQTC-------------KIKQQ
FPVPNQPVYN----QPVYNQP---VGAAGVPWMPAPQPPLNCPPGLEYLSQIDQILIHQQ
60
hPLSCR1
PfPLSCR
FDDREFVADYFMGFKLDFNNKYLILDASTELMKFTACEKSEFCYRNCLPKICIPMNMKIL hPLSCR1
IELLEV----LTGF--ETNNKYEIKNSFGQR-VYFAAEDTDCCTRNCCGPS-RPFTLRII

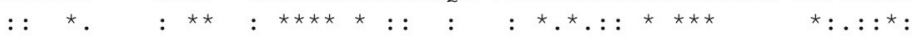
PEPLSCR
-SYGKEISKPDILMEKDCTCTF----LCFNRPTIKMYDFSNNNNKELIGTIKTPYRCCSY DNMGQEV----ITLERPLRCSSCCCPCCLQEIEIQ------APPGVPIGYVIQTWHPCLP
hPLSCR1
. $*{ }^{*}: * *^{*}: *$ * $:$ : * * * $:$ : $:$ *
PfPLSCR
NENLFDPSNNKIMYMDDTCCQMSILCPCPWGPFKYSNFFLRDANSKEKIAHLRK-EVPFL 234
hPLSCR1 KFTIQNEKREDVLKISGPCV----VCSCC-GDVDFE---IKSLDEQCVVGKISKHWIGIL 267
PfPLSCR KEVIRDIDNYTLDFEQVQNPEWKMMLLAFALFLDYMYYDRK---------- 275
hPLSCR1 REAFTDADNFGIQFPLDLDVKMKAVMIGACFLIDFMFFESTGSQEQKSGVW

C

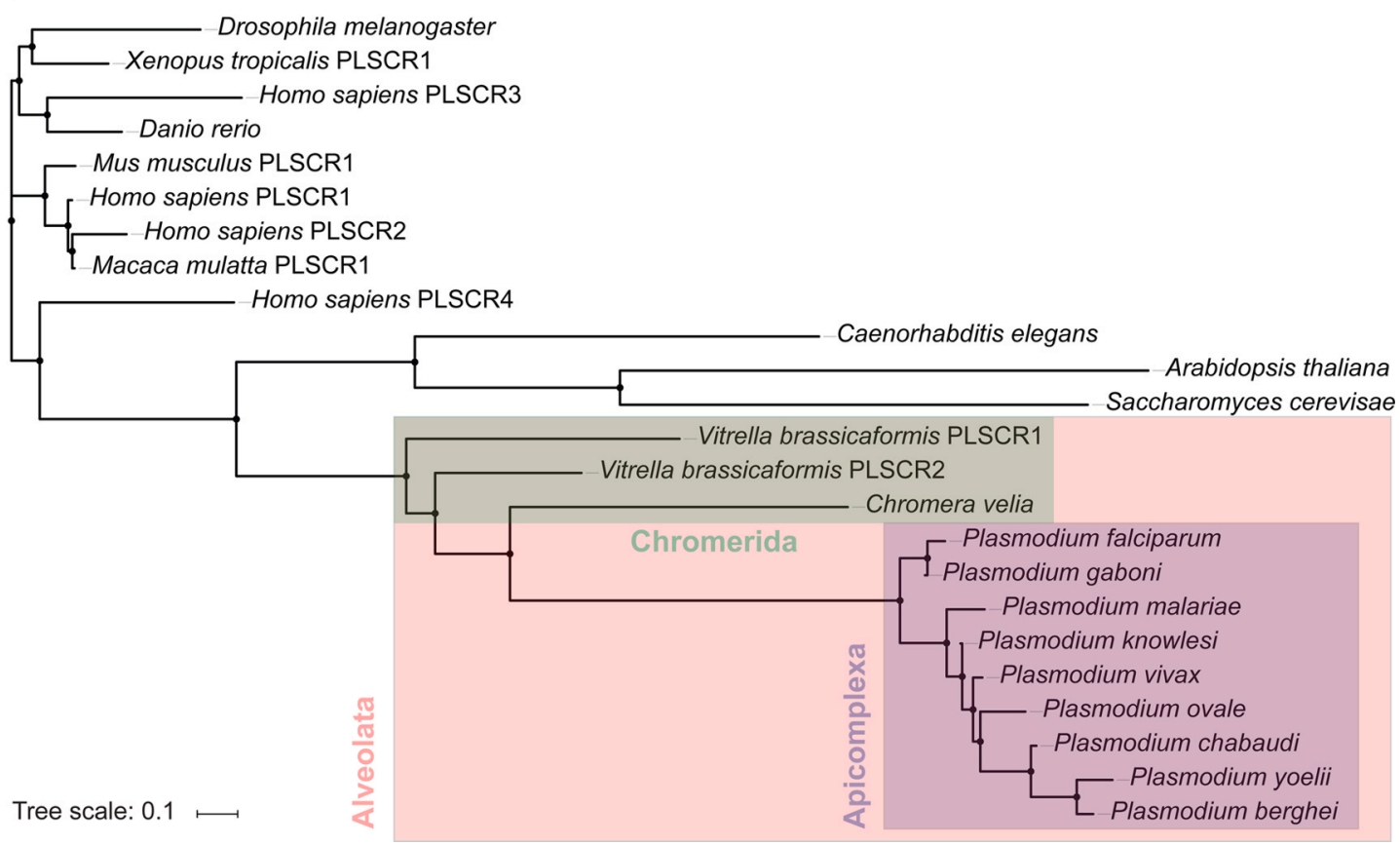

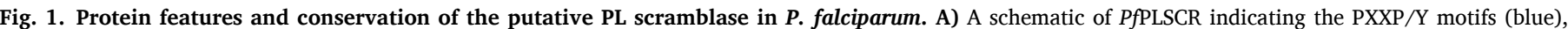

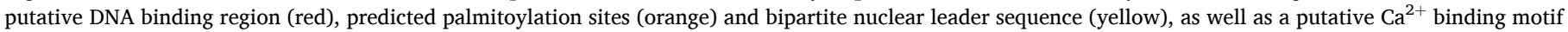

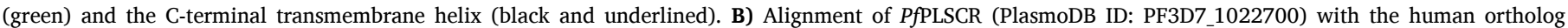

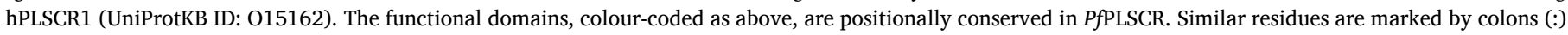

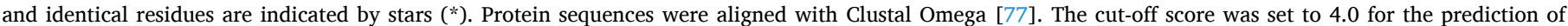

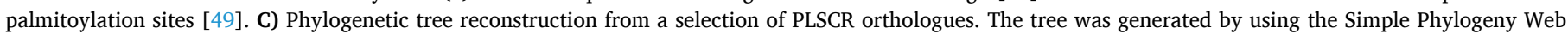

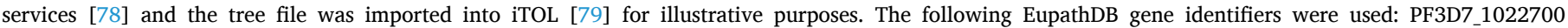

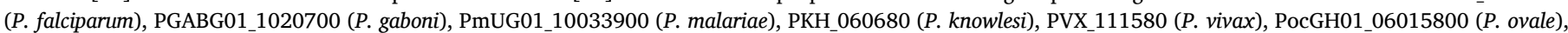

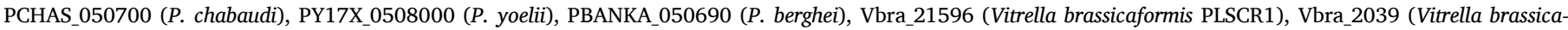

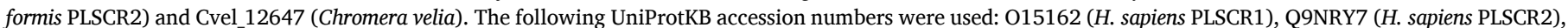

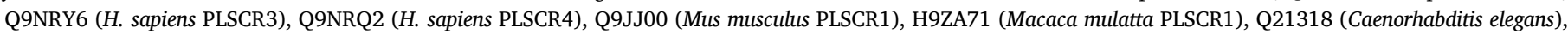
P47140 (Saccharomyces cerevisae), Q8IQD8 (Drosophila melanogaster), Q6NY24 (Danio rerio) and B5DDV8 (Xenopus tropicalis PLSCR1). 

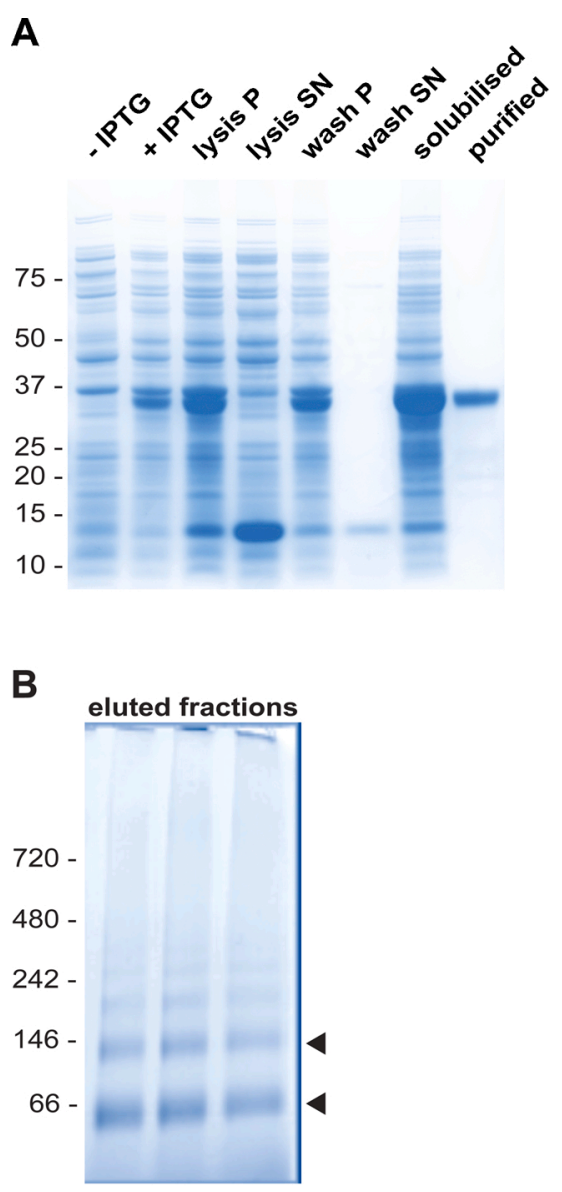

C
H. sapiens
M. musculus
M. mulatta
C. elegans
S. cerevisae
D. melanogaster
D. rerio
X. tropicalis
$P$. falciparum
V. brassicaformis
C. velia

D

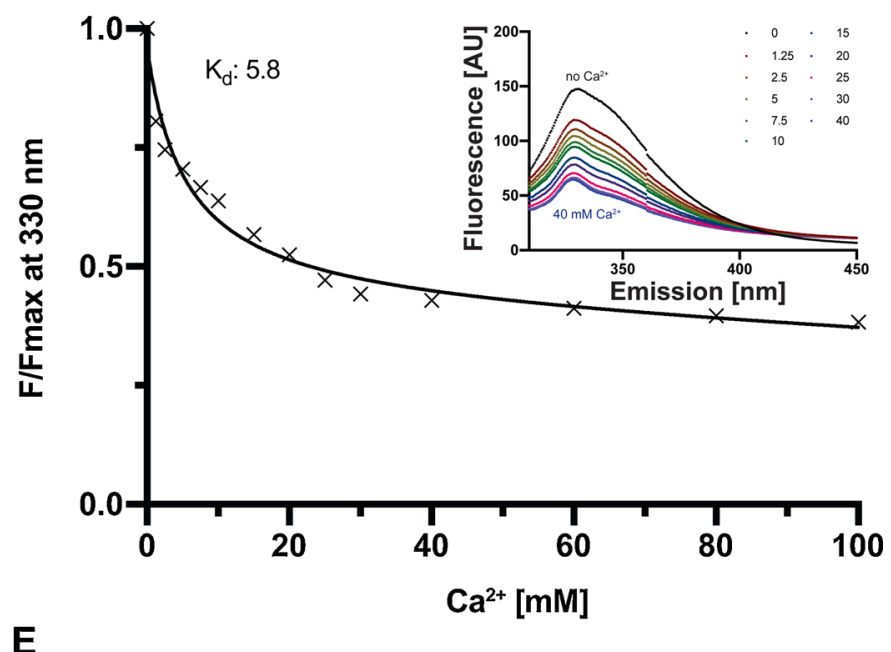

E

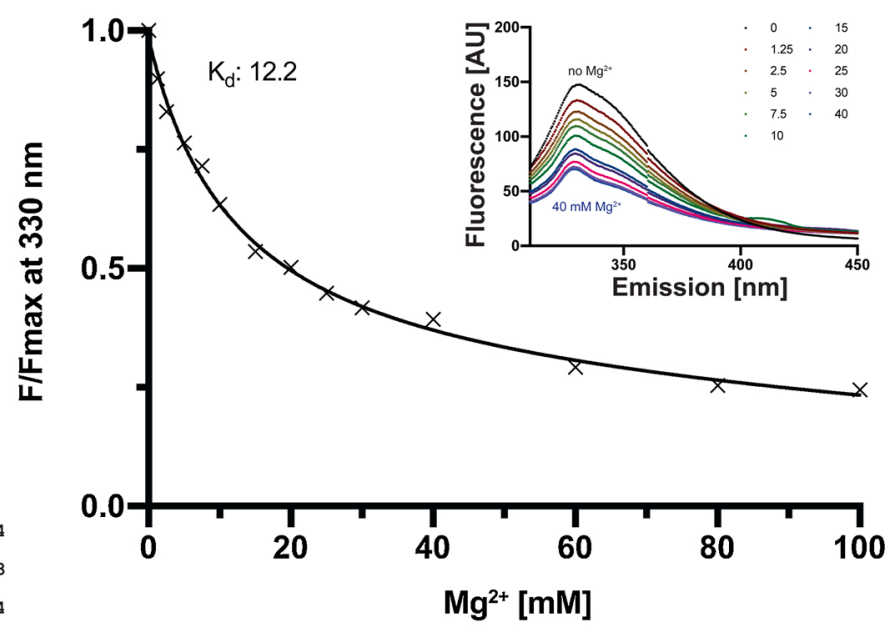

Fig. 2. PfPLSCR undergoes conformational changes in the presence of metal ions. A) SDS-PAGE of the expression and solubilisation of His::PfPLSCR. The purified recombinant protein runs at the predicted molecular weight of $\sim 37 \mathrm{kDa}$. Pellet and supernatant fractions are labelled as ' $\mathrm{P}$ ' and 'SN', respectively. B) BNPAGE of eluted fractions from the main peak reveals two protein species between the $66 \mathrm{kDa}$ and $146 \mathrm{kDa}$ protein markers (black arrow heads), indicating a possible dimerisation/oligomerisation of His::PfPLSCR. C) Alignment of the putative $\mathrm{Ca}^{2+}$ binding regions from different PLSCR1 orthologues. Residues in positions 1, 3, 5, 7, 9 and 12 of the EF-hand-like motif in hPLSCR1 have been proposed to octahedrally coordinate the calcium ion [24] and are shown in bold. Highly conserved residues are highlighted in blue, hydrophobic residues (positions 5 and 7) in green and the strictly conserved phenylalanine residues (position 9) are shown red. D \& E) Representative binding curves and calculated affinities (for the shown experiments) of recombinant PfPLSCR for $\mathrm{Ca}^{2+}$ and $\mathrm{Mg}^{2+}$ ions, respectively. The concentration-dependent decrease of fluorescence at $\lambda 330 \mathrm{~nm}$ is plotted as $\mathrm{F} / \mathrm{F}_{\max }$ with $\mathrm{F}=$ fluorescence measured in the presence of cations and $\mathrm{F}_{\max }=$ fluorescence under EGTA conditions. A selection of emission spectra is shown in the upper right corner.

chromatography. We therefore performed BN-PAGE to assess the potentially oligomeric state of His::PfPLSCR, which revealed two protein species suggestive of a dimeric and tetrameric form, respectively (Fig. 2B). Further attempts to determine the exact molecular mass of the protein species by size-exclusion chromatography multi-angle light scattering (SEC-MALS) delivered ambiguous results (data not shown), most likely due to the presence of different oligomeric species in the protein solution as implicated by the BN-PAGE of individual fractions.

\subsection{PfPLSCR is responsive to metal ions}

The hPLSCR1 protein contains a single EF-hand like $\mathrm{Ca}^{2+}$ binding loop, which has been shown to bind $\mathrm{Ca}^{2+}$ and other divalent cations in a co-ordinated manner, thus inducing conformational changes of the protein $[28,29,56]$. The unconventional $\mathrm{Ca}^{2+}$ binding motif of hPLSCR1 appears to be conserved in the parasite ortholog, including the residues in positions $1\left(\mathrm{Asp}^{240}\right), 3\left(\mathrm{Asp}^{242}\right)$ and $9\left(\mathrm{Phe}^{248}\right)$ (Fig. $2 \mathrm{C}$ ), all of which have been found to be crucial to the PL translocase activity of hPLSCR1 
A

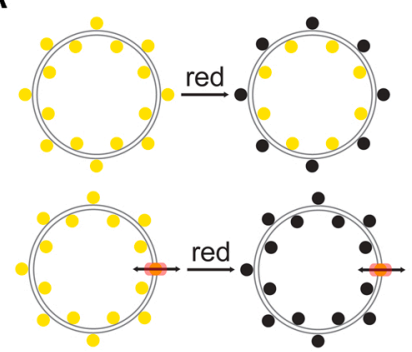

B

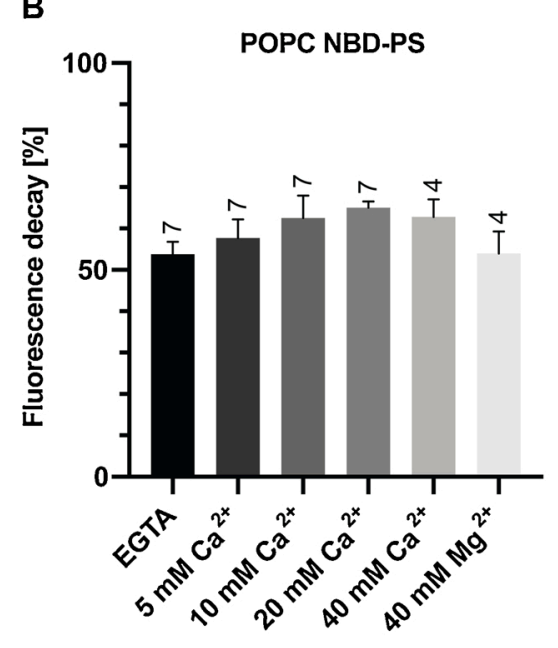

D

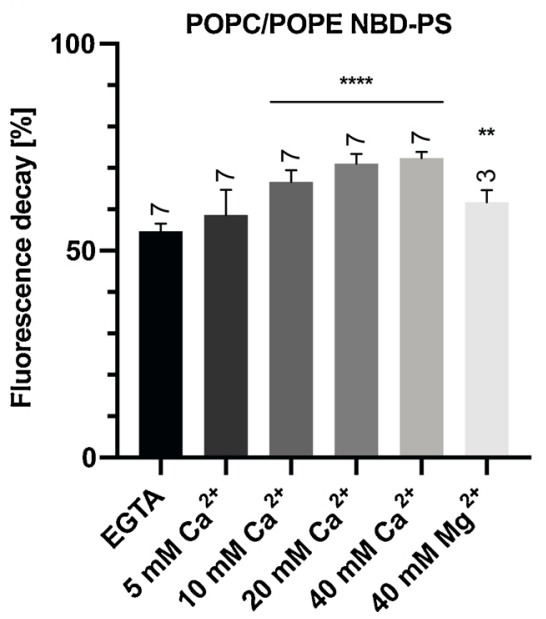

C

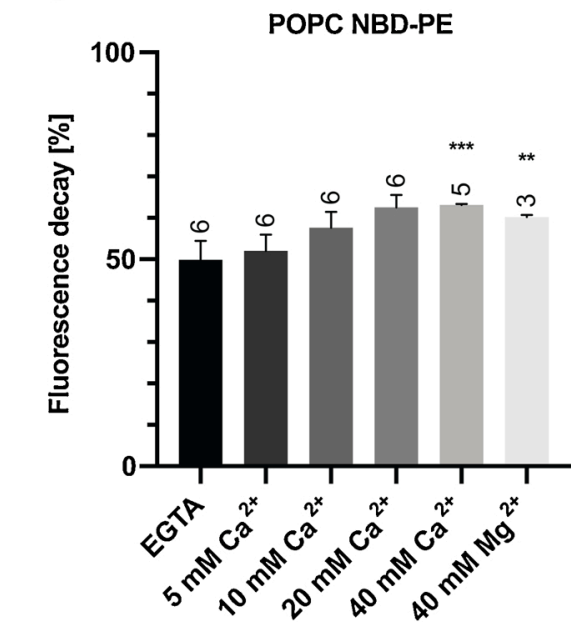

E

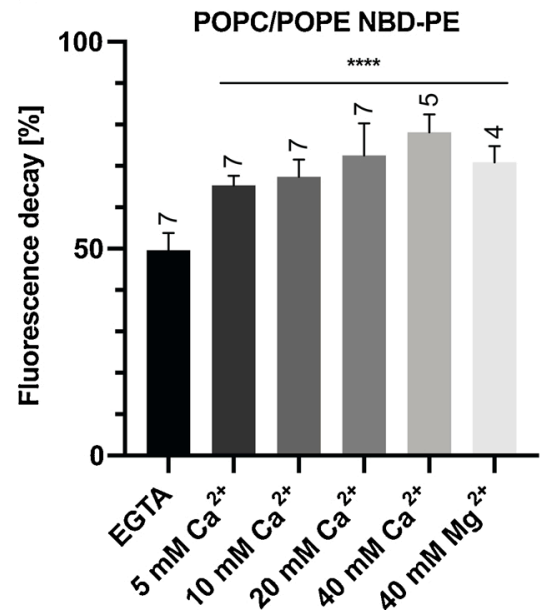

Fig. 3. PL scramblase activity of recombinant PfPLSCR in symmetrically labelled vesicles. A) Schematic of the 'scramblase assay'. B \& D) Transbilayer movement of NBDPS and C \& E) NBD-PE in proteoliposomes of different lipid compositions. Scramblase activity (mean $\pm \mathrm{SD}$ ) is presented as the percentage of fluorescence decrease at $500 \mathrm{~s}$. Number of independent experiments is indicated above the error bars. Statistical significance of differences between activities measured in the presence of EGTA and divalent cations was calculated using unpaired t-tests $(* * * *, p<0.0001$; ***, $p=$ $0.0001 ; * *, p \leq 0.005)$. 


\subsection{PfPLSCR mediates the transfer of PE and PS in reconstituted proteoliposomes}

To investigate the putative PL translocating properties of PfPLSCR, we reconstituted the recombinant protein into liposomes of different lipid compositions and performed 'scramblase assays' which allowed us to visualise and monitor PL translocase activity. Addition of sodium dithionate, which cannot cross the artificial membrane, to symmetrically labelled liposomes, reduces fluorescently labelled nitrobenzoxadiazole (NBD) lipids in the outer leaflet, resulting in an approx. $50 \%$ decrease of the initial fluorescence measured. Scrambling activity can be investigated by the ability of the putative PfPLSCR to transfer NBD lipids from the inner to the outer leaflet by which their exposure to the reducing environment will lead to a further decrease in fluorescence (Fig. 3A).

No conclusive PL translocase activity of recombinant His::PfPLSCR could be measured in proteoliposomes composed of PC only and NBD-PS (Figs. 3B \& S2A). Some activity, however, was observed if NBD-PE was used as a substrate, leading to a further $\sim 15 \%$ reduction in fluorescence and maximal catalytic function in the presence of $40 \mathrm{mM} \mathrm{Ca}^{2+}$ (Figs. 3C \& S2B). No PL scrambling could be detected under EGTA conditions, presumably rendering the protein inactive as a consequence of ineffective oligomerisation. Translocase activity could also be observed in the presence of $40 \mathrm{mM} \mathrm{Mg}^{2+}$ but with reduced efficiency and comparable to the fluorescence decrease at $10 \mathrm{mM} \mathrm{Ca}^{2+}$ (Figs. 3C \& S2B). Respective liposomes without PfPLSCR displayed no further decrease in fluorescence beyond the $50 \%$ margin as expected (dotted lines in Fig. S2A-B).

In attempts to mimic the PL composition of the parasite's membranes, an overall greater catalytic activity was noted in reconstituted proteoliposomes composed of PC and PE (70:30\%), with a further decrease in fluorescence of maximally $\sim 20 \%$ for vesicles labelled with NBD-PS (Figs. 3D \& S2C) and $\sim 30 \%$ for NBD-PE containing proteoliposomes (Figs. 3E \& S2D). Phospholipid scrambling activity was enhanced by the increase of metal ion concentrations, reaching maximal activity at $20-40 \mathrm{mM} \mathrm{Ca}^{2+} . \mathrm{Mg}^{2+}$ activated PL scrambling could also be observed in these vesicles but once again with lower efficiency. Importantly, no $\mathrm{Ca}^{2+}$ induced fluorescence decay (beyond the $50 \%$ margin) was observed for protein-free liposomes composed of PC and PE, excluding the possibility of PE-mediated bilayer destabilisation and liposome 'leakiness' (Fig. S2E-F).

These data strongly suggest a metal ion dependent PL translocase function for PfPLSCR with a preference for calcium ions and NBD-PE as a substrate as indicated by maximal activity under these conditions. A recent lipidomic analysis of the total lipid composition in asexual blood stage parasites showed that PC and PE accounted for $50 \%$ of the total lipid content [3]. Inclusion of PE in reconstituted proteoliposomes resulted in an overall increase of His::PfPLSCR mediated PL translocase efficiency, likely due to a more physiological environment allowing for optimal protein function.

\subsection{Generation of an inducible PfPLSCR knock out line}

Having demonstrated PL translocase activity for PfPLSCR, we undertook a genetic approach to characterise the protein in $P$. falciparum parasites. We used a previously published genetic system [33] that modifies the endogenous Pfplscr locus by single-crossover homologous recombination using the selection-linked integration (SLI) strategy [40] and generated a conditional knock out (cKO) parasite line expressing C-terminally triple-HA tagged full length PfPLSCR (hereafter referred to as PfPLSCR-HA) under its endogenous promoter. Two synthetic loxPint modules [34] were inserted into the targeting plasmid, allowing for DiCre-mediated excision of the sequence encoding the C-terminal region Lys $^{217}$-Lys $^{275}$ of the protein in the optimised DiCre recombinase expressing B11 parasite line [39] (Fig. 4A). Transgenic parasites were retrieved after 2 weeks of initial WR99210 selection and a clonal population of integrant parasites was obtained 7 days after neomycin selection using SLI [40]. Successful modification of the Pfplscr locus was confirmed by diagnostic PCR (Fig. 4B) and Western blot analysis giving rise to $\mathrm{a} \sim 37 \mathrm{kDa}$ protein (doublet) band as predicted for endogenously tagged PfPLSCR-HA (Fig. 4C). The growth rate of PfPLSCR-HA expressing parasites was similar to that of the parental B11 line (Fig. 4D), suggesting no negative side effects of the modification to parasite viability.

\subsection{PfPLSCR-HA localises to different membranous compartments throughout asexual development}

The Pfplscr gene is transcribed throughout the intra-erythrocytic life cycle but is maximally expressed in late schizonts stages $(40-48 \mathrm{~h}$ post invasion, www.PlasmoDB.org). Immunoblot analysis of parasite protein lysates prepared at different timepoints post invasion confirmed the expression of PfPLSCR-HA across the asexual life cycle (Fig. 4C). Immunofluorescence analysis revealed the localisation of the epitopetagged protein to the periphery of individual merozoites in late schizonts as suggested by co-localisation with the merozoite surface protein MSP1 (Fig. 4E). The protein also localised to a perinuclear region in the early ring stages as evident by partial co-localisation with the ERresident protein signal peptide peptidase [44], and redistributed to the cytoplasm and vesicular foci in early trophozoite and schizont stages (Fig. 4E). Several attempts to verify the localisation of PfPLSCR-HA at the plasma membrane and other membranous compartments by immuno-EM proved inconclusive, likely due to the inaccessibility of the HA epitope being buried in the membrane. A solubility analysis, however, confirmed a tight membrane association of PfPLSCR-HA throughout the asexual life cycle (Fig. 4F) supporting a possible PL translocase function in the membrane bilayer.

\subsection{PfPLSCR is not essential for erythrocyte invasion and asexual development}

Investigations into the nature of the C-terminal transmembrane helix of hPLSCR1 confirmed its function in inserting the protein into the bilayer of reconstituted liposomes and revealed an additional role in coordinating the metal ion, thus influencing scramblase activity [25, 57]. Two cholesterol-binding sites, composed of $\mathrm{Ala}^{291}, \mathrm{Phe}^{298}, \mathrm{Leu}^{299}$ and $\mathrm{Leu}^{299}$, $\mathrm{Phe}^{302}, \mathrm{Glu}^{306}$, respectively have also been identified and proposed to stabilise the C-terminal transmembrane helix in the phospholipid bilayer. The unusual Asp ${ }^{301}$ residue has further been suggested to play a role in scramblase activity [58].

To explore the specific role of PfPLSCR in parasite development, rapamycin induced, DiCre-mediated excision of the 'floxed' sequence in the PfPLSCR-HA line was anticipated to generate a mutant scramblase protein lacking the putative NLS, $\mathrm{Ca}^{2+}$-binding motif and C-terminal trans-membrane helix Lys $^{217}$-Lys $^{275}$ which includes the conserved $\mathrm{Asp}^{268}$ residue) and therefore PL translocase activity, which we hypothesised to be important for host cell invasion and/or intraerythrocytic development (Fig. 5A). Diagnostic PCRs confirmed successful excision of the locus compared to DMSO-treated PfPLSCR-HA parasites (Fig. 5B). Excision also removed the neo- $R$ gene, enabling expression of the C-terminally truncated scramblase protein in fusion with GFP as evidenced by Western blot analysis (Fig. 5C-D). Live-cell fluorescence of the remaining GFP fusion protein was weak and not clearly distinguishable from background fluorescence but as predicted, plasma membrane localisation in merozoites was no longer observed as visualised by immuno-fluorescence analysis using anti-GFP antibodies in the conditional KO parasites (Fig. 5D). However, no significant growth or invasion defect could be detected for parasites expressing truncated PfPLSCR in the induced KO compared to full length PfPLSCRHA in mock-treated parasites over a total period of 3 cycles as analysed by flow cytometry (Fig. 5E) or light microscopy of Giemsa-stained blood smears (data not shown). These findings suggest PfPLSCR is dispensable for erythrocyte invasion and asexual parasite development. 
A

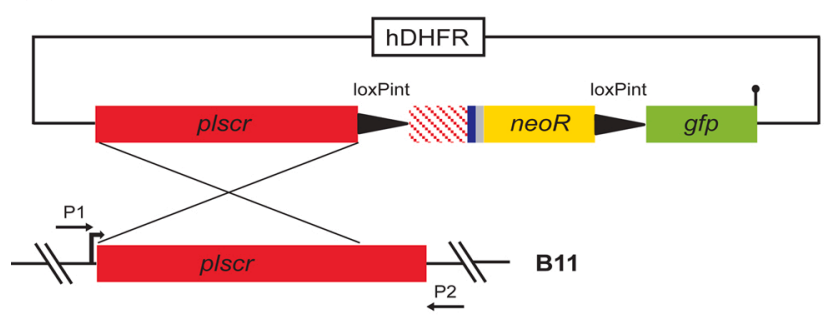

PARL PFPLSCR ${ }_{\text {loxP }}$

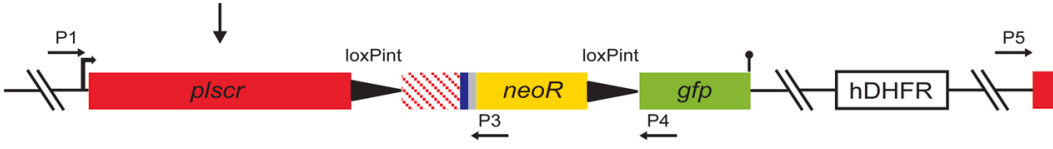

\section{B}
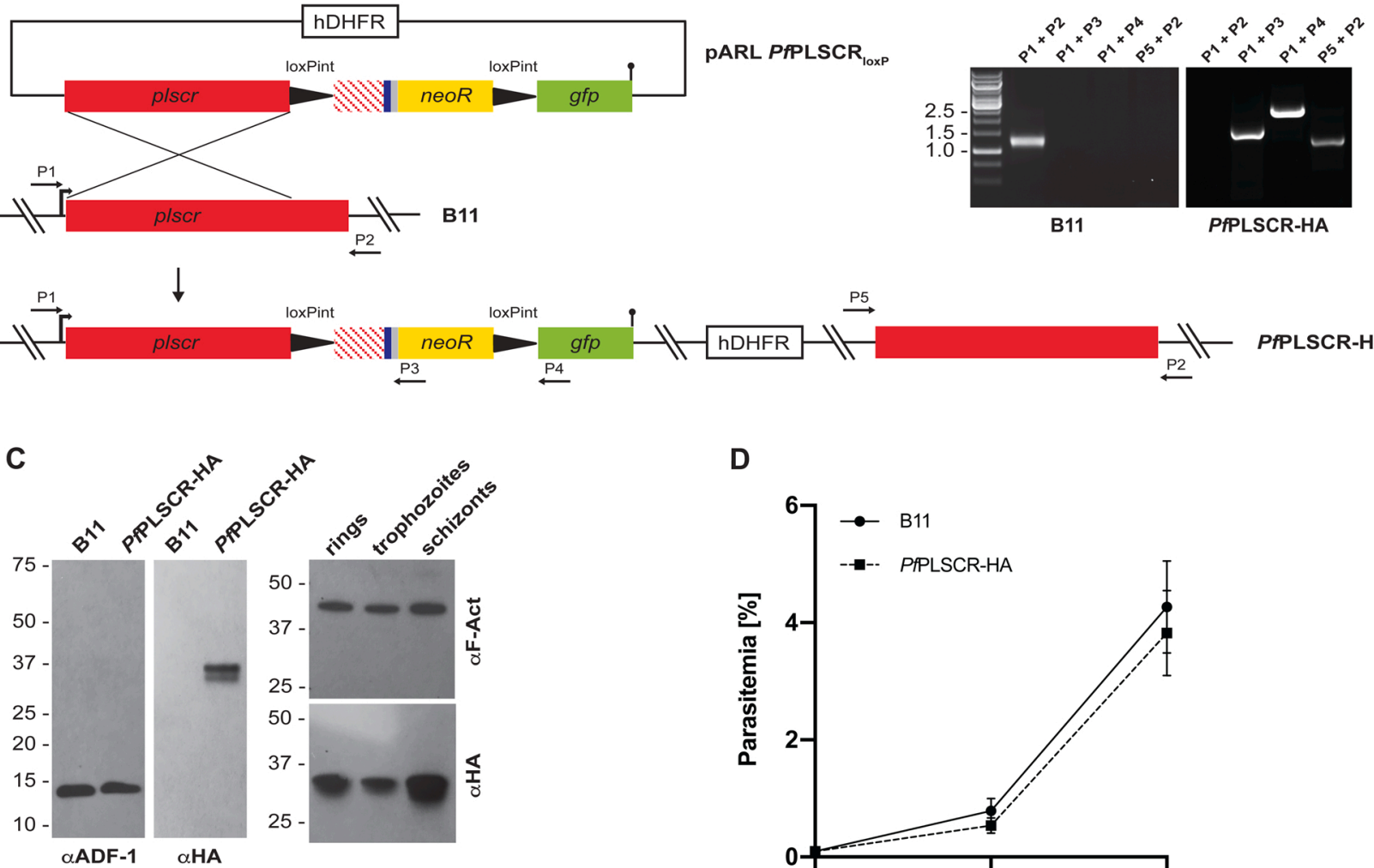

E
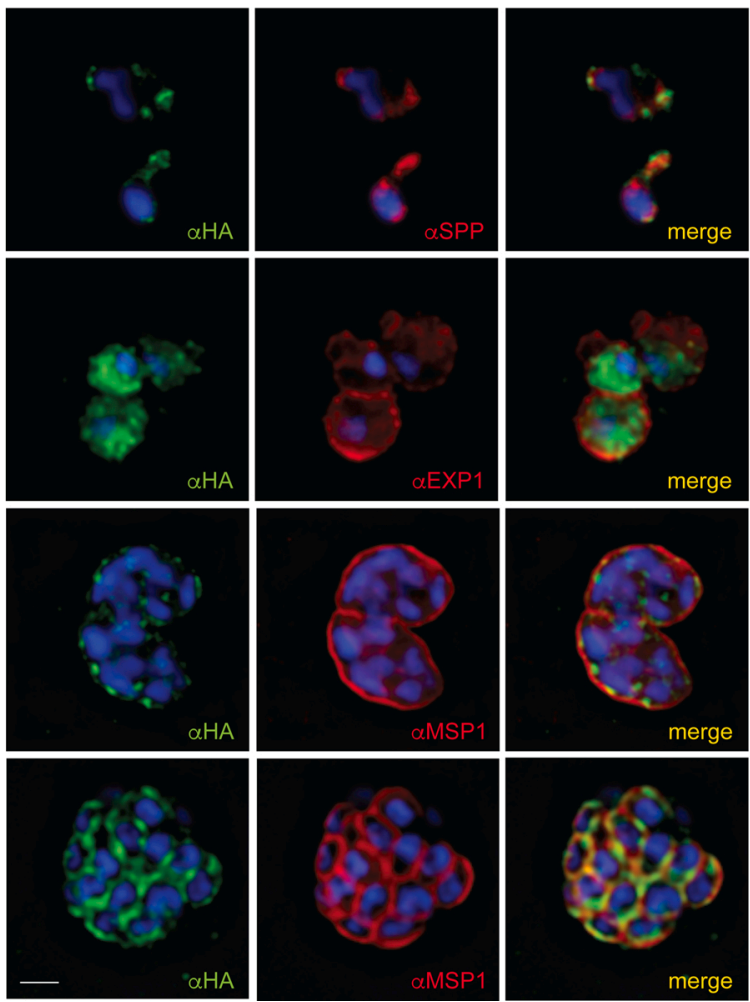

D
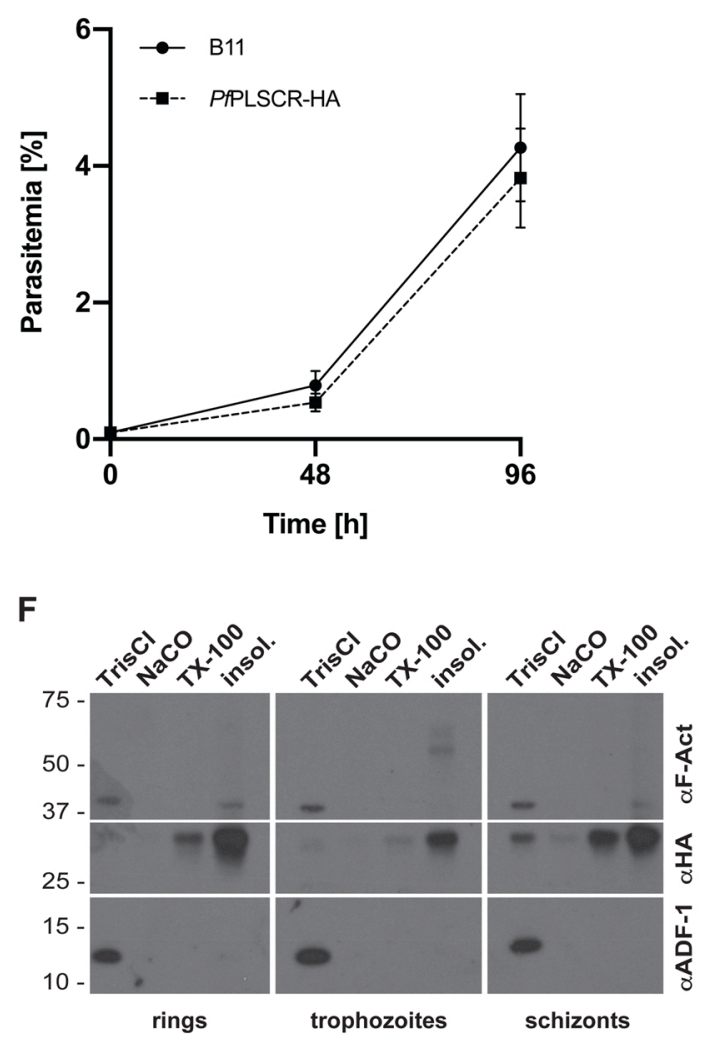
Fig. 4. PfPLSCR-HA is expressed throughout the asexual life cycle and localises to membranous structures in the parasite. A) Schematic of the strategy to generate the conditional KO line PfPLSCR-HA via SLI mediated single-crossover homologous recombination. The targeting plasmid contains the Pfplscr homology region (red), followed by two synthetic loxPint modules flanking the recodonised Pfplscr sequence (red stripes) with a triple-HA tag (blue), T2A skip peptide (gray) and neomycin resistance cassette (yellow). The GFP coding sequence (green) is followed by a stop codon (black bar). Numbered arrows indicate primers used for genotyping. B) Diagnostic PCR confirms successful integration into the Pfplscr locus by amplification of three distinctive fragments at the expected size (1585, 2455 and $1182 \mathrm{bp}$ ) and loss of the wild-type band (1396 bp) in the PfPLSCR-HA parasite line. C) Western blot analysis gives rise to a $\sim 37 \mathrm{kDa}$ protein band as predicted for PfPLSCR-HA. The endogenously tagged protein is expressed throughout the intra-erythrocytic life cycle as indicated by the different parasite stages (hours post invasion): rings $(4 \pm 2 \mathrm{~h})$, trophozoites $(20 \pm 4 \mathrm{~h})$ and schizonts $(44 \pm 4 \mathrm{~h})$. Anti-ADF (actin depolymerising factor) and anti-F-Actin antibodies are used as loading controls. D) Growth curves of parental B11 and PfPLSCR-HA expressing parasites over two cycles. Parasitemias (as quantified by flow cytometry) were averaged from at least three biological replicates using blood from different donors and are presented as mean \pm SD. E) Immunofluorescence analysis of PfPLSCR-HA expressing parasites. The upper panel depicts two ring-stage parasites and co-labelling with anti-SPP (signal peptide peptidase) antibodies as an ER marker. Anti-EXP1 (exported protein 1) delineates the PVM surrounding three trophozoites in a multiply infected red blood cell. Lower two panels show deconvolved co-labelling with antibodies against MSP1 (merozoite surface protein 1) in late blood stage parasites. PfPLSCR-HA co-localises with MSP1 at the plasma membrane in late schizonts. Parasite nuclei are stained with DAPI (blue). Scale bar $=1 \mu \mathrm{M}$. F) PfPLSCR-HA remains strongly membrane-associated throughout the intra-erythrocytic life cycle as suggested by sequential extraction of the protein and its presence in the TX-100 insoluble fraction. The blot was cut and probed with anti-ADF as a control for soluble ADF protein and anti-F-Actin as a control for partially membrane-bound parasite actin.

To exclude any residual activity of the truncated PfPLSCR protein, we attempted a full KO of the Pfplscr locus using CRISPR-Cas9 by replacing the gene with a super folder GFP (sfGFP) expression cassette (Fig. 6A). Different combinations of the plasmid harbouring the Cas9 expression cassette [36], as well as one of three different guide RNAs tested, were co-transfected with the donor plasmid. One plasmid combination, containing guides 952497 and 952530 , both in close proximity to the 5' targeting flank, resulted in successful parasite recovery after 5 weeks post transfection as evident by sfGFP positive parasites (Fig. 6D). Replacement and loss of the Pfplscr gene with the sfGFP expression cassette was further confirmed by diagnostic PCR (Fig. 6B). A growth analysis of 3D7 wild-type versus PfPLSCR KO parasites revealed no significant differences (Fig. 6C), further corroborating the redundancy of the PL scramblase protein in Plasmodium spp. asexual development.

\subsection{PfPLSCR is expressed in gametocytes}

Transcriptomic data suggests that PfPLSCR is also transcribed in the sexual stages of $P$. falciparum (www.PlasmoDB.org). A recent report on the role of a female gametocyte-specific ATP-binding cassette transporter (gABCG2) in regulating the metabolism of neutral lipids [59] prompted us to investigate the expression of PfPLSCR-HA in gametocytes. Immunofluorescence analysis of stage III-IV gametocytes revealed different localisation patterns of the epitope-tagged protein. Localisations ranged from an intracellular distribution with more or less pronounced vesicular foci (Fig. 7A), to a peripheral localisation as indicated by co-labelling with Pfs16, a gametocyte specific protein of the PVM (Fig. 7A, last panel) and GAP45 (glideosome associated protein, Fig. 7B), a component of the IMC (inner membrane complex), which has also been identified in gametocyte stages [60]. As the PVM, parasite plasma membrane and IMC cannot be distinguished at this resolution, a more detailed investigation at the ultrastructural level will be necessary to determine the exact location of PfPLSCR-HA at the parasite's periphery in gametocytes. However, imaging and immunoblot analysis confirm the expression of PfPLSCR-HA in gametocytes (Fig. 7C) and, though negligible for asexual development, suggests a possible role of the PL translocase in the sexual development of Plasmodium spp. parasites.

\section{Discussion}

In this study, we identified and characterised a putative PL scramblase in the malaria parasite P. falciparum, which we hypothesised to play a role in erythrocyte invasion and/or asexual parasite development. A sequence comparison with hPLSCR1 revealed a shorter proline-rich Nterminus in PfPLSCR but the functional domains of the C-terminal portion appear to be conserved (Fig. 1B). Conservation in the $\mathrm{C}$ terminus is consistent with observations made for the four hPLSCR homologs and various other PLSCR orthologs found throughout the animal kingdom (reviewed in [52]). The proline-rich domain (PRD) has been shown to mediate the subcellular localisation, $\mathrm{Ca}^{2+}$-dependent aggregation and therefore PL scrambling activity of hPLSCR1. The absence of this domain in hPLSCR2 renders the protein functionally inactive as a PL translocase but can be restored by addition of the hPLSCR1 PRD to the N-terminus of hPLSCR2 [28]. The PXXP and PPXY motifs found in hPLSCR1, 3 and 4 are further thought to serve as potential binding sites for proteins containing SH3 and WW domains [18], scaffolds which recognize proline-rich regions in a variety of signaling and structural proteins.

Despite a shorter PRD and only one potential PXXP motif, intrinsic tryptophan fluorescence measurements on recombinant PfPLSCR reveal $\mathrm{Ca}^{2+}$ and $\mathrm{Mg}^{2+}$ induced conformational changes, suggesting a functional preservation of the $\mathrm{Ca}^{2+}$ binding site. Residues in positions 1, 3, 5, 7, 9 and 12 of the EF-hand-like motif in hPLSCR1 have been proposed to octahedrally coordinate the $\mathrm{Ca}^{2+}$ ion [24] and are functionally conserved in the $\mathrm{Ca}^{2+}$ binding region of the parasite ortholog, with the exception of the terminal Val residue (Fig. 2C). Calculated dissociation constants in the mM range (Fig. 2D-E) indicate a low affinity for the divalent cations tested but are in accordance with the established values for hPLSCR1 and hPLSCR2 [28]. BN-PAGE of His::PfPLSCR purified in the presence of $0.5 \mathrm{mM} \mathrm{Ca}^{2+}$ suggests the formation of dimeric and/or oligomeric species of the protein (Fig. 2B), supporting the notion of $\mathrm{Ca}^{2+}$ mediated oligomerisation [28] and functionality of the reduced PRD region in PfPLSCR. Oligomer formation has also been observed for erythrocyte hPLSCR1 [61] and a Cryo-EM study revealed the dimeric organisation of TMEM16 phospholipid scramblase from Nectria haematococca [32]. It should be noted that omission of $\mathrm{Ca}^{2+}$ in the purification of His::PfPLSCR reduced aggregation during size-exclusion chromatography but rendered intrinsic fluorescent measurements and PL scramblase assays inconclusive.

Maximal PL translocase activity of recombinant PfPLSCR was measured in the presence of $40 \mathrm{mM} \mathrm{Ca}^{2+}$ and using NBD-PE as a substrate in proteoliposomes composed of POPC and POPE (Figs. 3E \& S2D), thus mimicking the lipidic environment of the parasite's membranes [3]. These experimental conditions resulted in an approx. $80 \%$ decrease of initial NBD-fluorescence recorded (50\% quenching of fluorophores in the outer leaflet upon sodium dithionate treatment and a further $30 \%$ decrease due to translocase activity), which is well in line with established maximal activities of $80-85 \%$ in other proteins with PL scramblase function [32,62-64]. The high $\mathrm{Ca}^{2+}$ concentration needed to activate recombinant His::PfPLSCR, however, by far exceeds the physiological concentration of $\mathrm{Ca}^{2+}$ in Plasmodium spp. parasites [65]. Reported PL translocation rates for hPLSCR1 appear to be negligible, most likely due to the use of NBD-PC as a substrate and/or insufficient reconstitution of recombinant hPLSCR1 into liposomes [24,27-29]. Supporting this notion, substrate preference has been observed in hPLSCR1 with higher catalytic activity for NBD-PE over NBD-PC [30] and in mitochondrial hPLSCR3, which preferentially translocates NBD-PE and NBD-PS, apart from cardiolipin [66]. The exact mechanism 
A

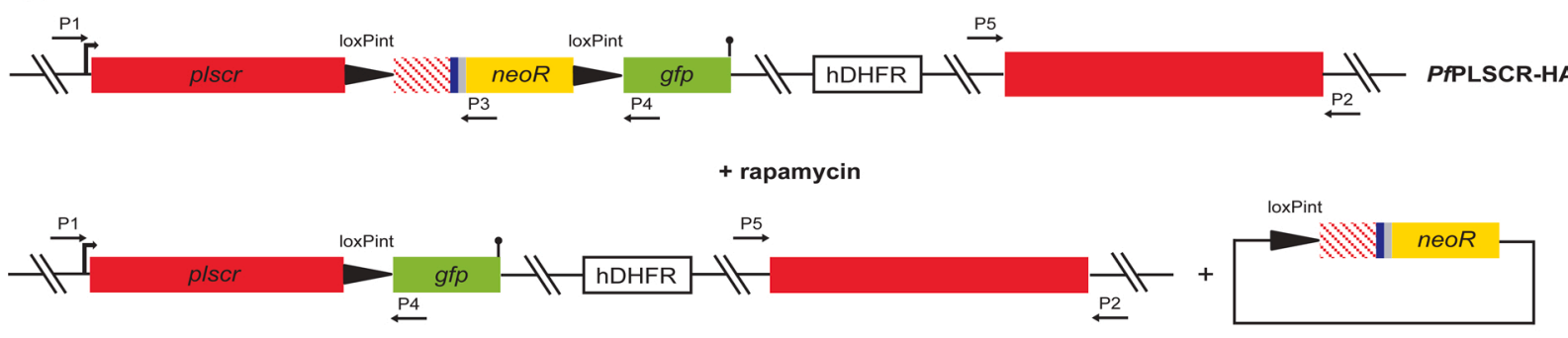

B

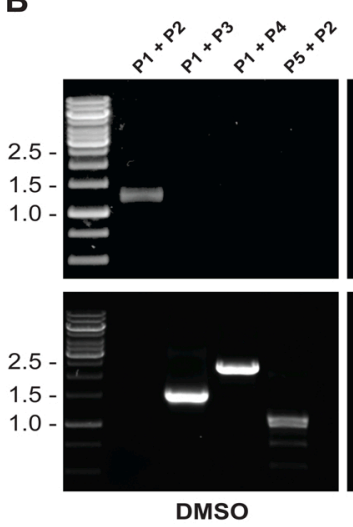

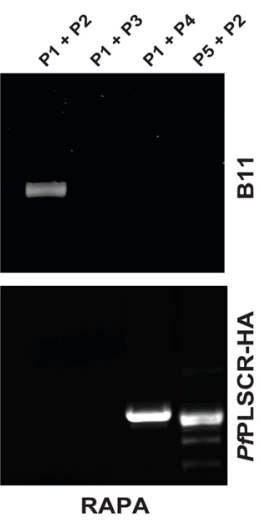

C

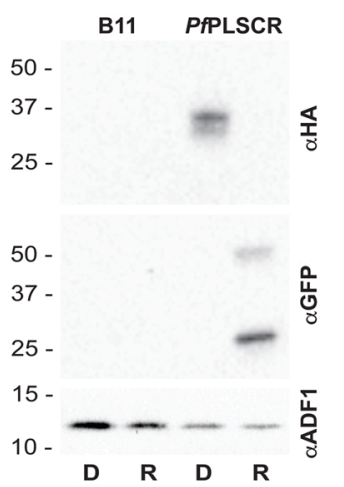

D

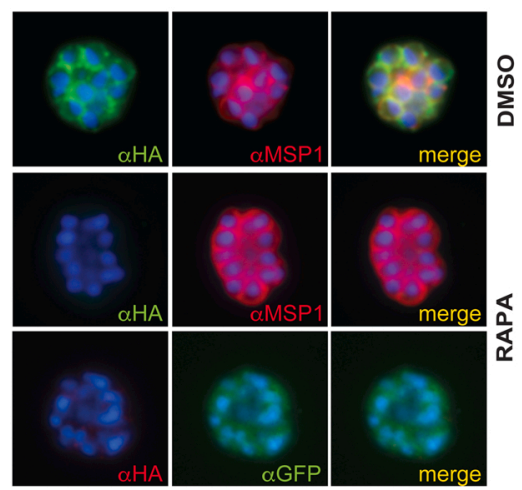

E

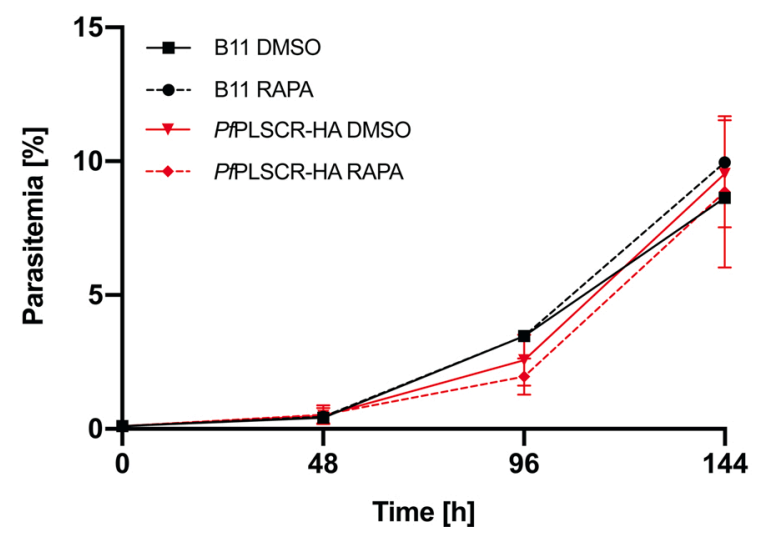

Fig. 5. PL scrambling is not essential for red blood cell invasion and intra-erythrocytic parasite development. A) Schematic of rapamycin induced and DiCremediated excision of the floxed sequence in PfPLSCR-HA. B) Diagnostic PCR confirms successful excision of the locus compared to DMSO-treated PfPLSCR-HA parasites. C) The C-terminally truncated PfPLSCR protein is expressed as a GFP fusion protein at the expected molecular weight of $\sim 53 \mathrm{kDa}$ in rapamycin-treated parasites. The $\sim 27 \mathrm{kDa}$ band is characteristic for GFP only. Anti-ADF antibodies are used as a loading control. D) Plasma membrane association is lost in parasites expressing the truncated PfPLSCR-GFP fusion protein, which distributes throughout the parasite cytosol. E) Growth curves of rapamycin and DMSO-treated parasites over three cycles. Parasitemias were averaged from at least three biological replicates using blood from different donors and are presented as mean \pm SD.

of phospholipid translocation by the members of this PL scramblase family, however, remains to be resolved.

The generation of a conditional PfPLSCR KO line enabled the investigation of triple-HA tagged full length PfPLSCR in $P$. falciparum parasites. Expression of PfPLSCR-HA under the endogenous promoter was observed throughout asexual parasite development with maximal expression in late schizont stages (Fig. 4C). Western blot analysis revealed $\mathrm{a} \sim 37 \mathrm{kDa}$ HA-positive protein doublet, mainly present in late schizont stage protein samples, implying a possible modification of PfPLSCR in the parasite. Human PLSCR1 was found to be phosphorylated by PKC $\delta$ in kinase assays [55] and differentially phosphorylated in Jurkat cells with a shift from serine phosphorylation in unstimulated to threonine phosphorylation in apoptotic cells [67]. The authors further proposed an increased scramblase activity upon activation of PKC $\delta$ and phosphorylation of $\mathrm{Thr}^{161}$. However, no phospho-peptides could be identified for PfPLSCR in a recent phospho-proteomic study of schizont samples [68], indicating a different nature of protein modification likely exists for PfPLSCR.

PfPLSCR-HA localises to different membranous compartments in asexual parasites (Fig. 4E-F). The redistribution of PfPLSCR-HA from a perinuclear association in rings, to a more diffuse and vesicular dispersion in trophozoites and plasma membrane localisation in late schizonts resembles the observations made for hPLSCR1 which is trafficked to the plasma membrane of fibroblasts in the presence of palmitoylation but is found in the cytoplasm and nucleus of cells expressing mutant PLSCR lacking the five palmitoylation residues in the cysteinerich motif ${ }^{184}{ }^{C C C P C C}{ }^{189}$ [22]. Mutation of the corresponding palmitoylation sites in hPLSCR3 and hPLSCR4 also results in nuclear 
A
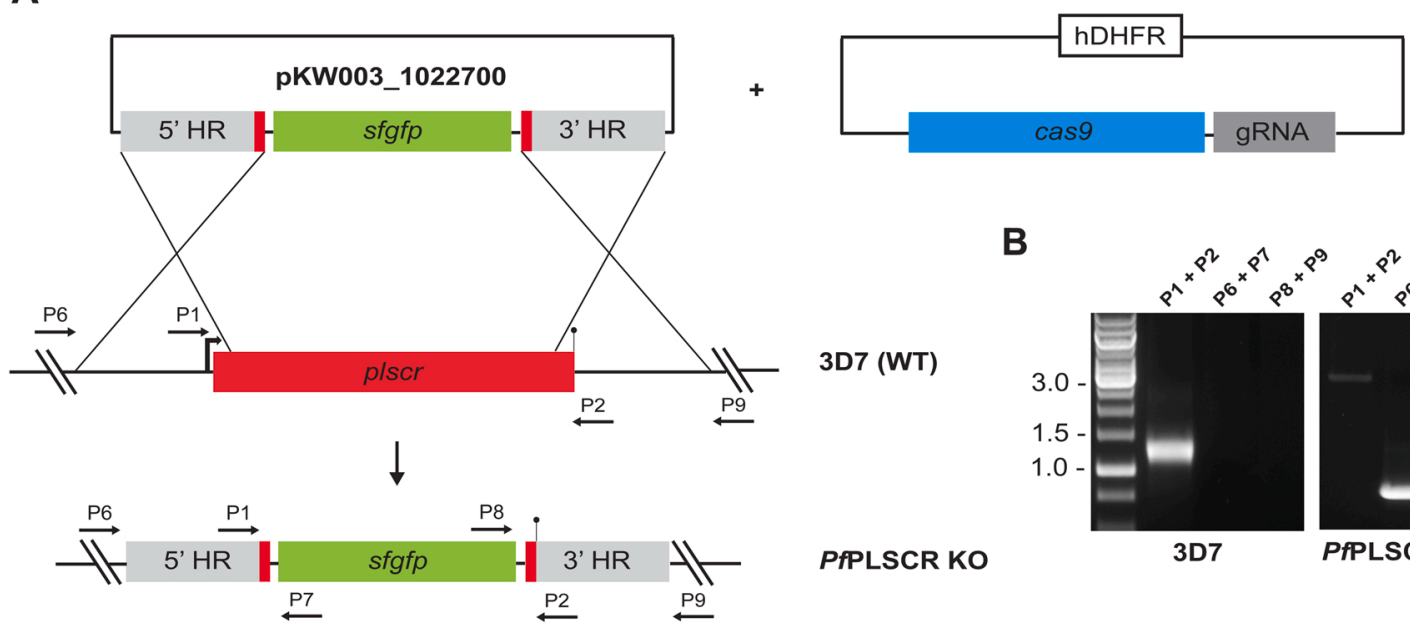

$3 \mathrm{D} 7$ (WT)

C

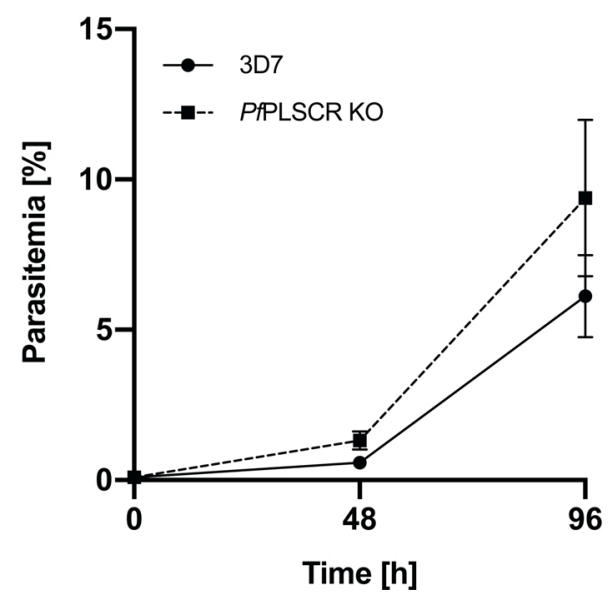

D

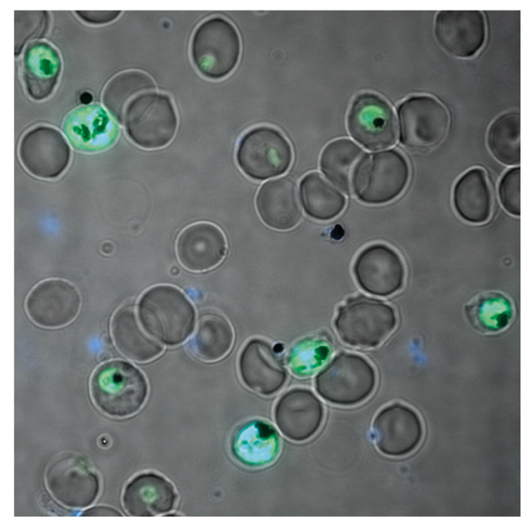

Fig. 6. A full gene KO in 3D7 parasites supports the redundancy of PfPLSCR in asexual parasite biology. A) Schematic of the CRISPR-Cas9 mediated KO

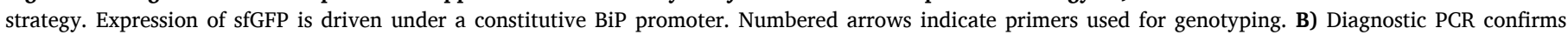

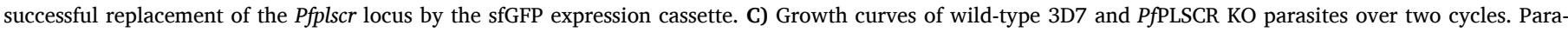

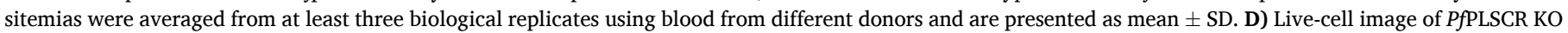
parasites expressing sfGFP throughout asexual parasite development. Parasite nuclei are stained with DAPI (blue).

redistribution $[69,70]$. Nuclear import of hPLSCR1 and hPLSCR4 has been proposed to be mediated by a minimal non-classical nuclear leader sequence (NLS) and to occur via the $\alpha / \beta$ importin pathway [23,70,71]. A subsequent study demonstrated transcriptional regulation of the IP3R1 receptor by hPLSCR1 [21], further supporting a potential nuclear function of hPLSCR1. PfPLSCR possesses a predicted bi-partite NLS (Fig. 1B) and has been identified as a putative palmitoyl protein [72], concordant with the prediction of two palmitoylation sites at ${ }^{172} \mathrm{CC}^{173}$ and ${ }^{194} \mathrm{CC}^{195}$ (http://csspalm. biocuckoo.org [49]). While this needs to be experimentally proven, stage-specific palmitoylation of PfPLSCR could explain the differential localisation and presence of the discrete doublet for PfPLSCR-HA.

The biochemical verification of PL translocase activity, maximal expression of PfPLSCR-HA in late schizont stages, the localisation to the plasma membrane in merozoites and the unique conservation of this (otherwise ubiquitously present) protein in Plasmodium spp. but no other apicomplexan parasite reinforced our hypothesis of a role for PfPLSCR in the infection of the host erythrocyte. Two different gene knockout approaches, however, revealed no invasion or intra-erythrocytic growth defect in the generated knockout lines (Figs. 5E \& 6 C). Plasma membrane association of PfPLSCR is no longer observed in late stage parasites expressing the mutant PfPLSCR GFP fusion protein in the conditional KO line (Fig. 5D) and supports an anchoring role of the predicted C-terminal transmembrane region in PfPLSCR. Complete deletion of the gene in full PfPLSCR KO parasites (Fig. 6), as well as a recent large-scale mutagenesis screen using the piggyBac transposon in $P$. falciparum [73], confirm the redundancy of PfPLSCR in asexual parasite development. It should be noted that parasite lines were maintained under optimal growth conditions and it remains to be established whether nutrient limited conditions with reduced or modified lipid sources may influence parasite development and/or life cycle progression in the deficient PfPLSCR lines. A search of the PlasmoGEM database (https://plasmogem.sanger.ac.uk/), however, also affirmed the rodent ortholog PBANKA_0506900 in P. berghei to be dispensable for intra-erythrocytic development, further corroborating our results.

While the biological function and preservation of PfPLSCR remains elusive, expression of PfPLSCR-HA in gametocytes (Fig. 7) may indicate a role in other stages of the parasite lifecycle or transitions between them. Another intriguing finding is the presence of orthologs in the closely related alveolates Chromera velia and Vitrella brassicaformis. Though the algae have not been found to sexually reproduce, they do form elongated zoospores with two heterodynamic flagella of unknown function [55], which resemble exflagellated male gametocytes of P. falciparum parasites. Recent reports on the role of a female 
A

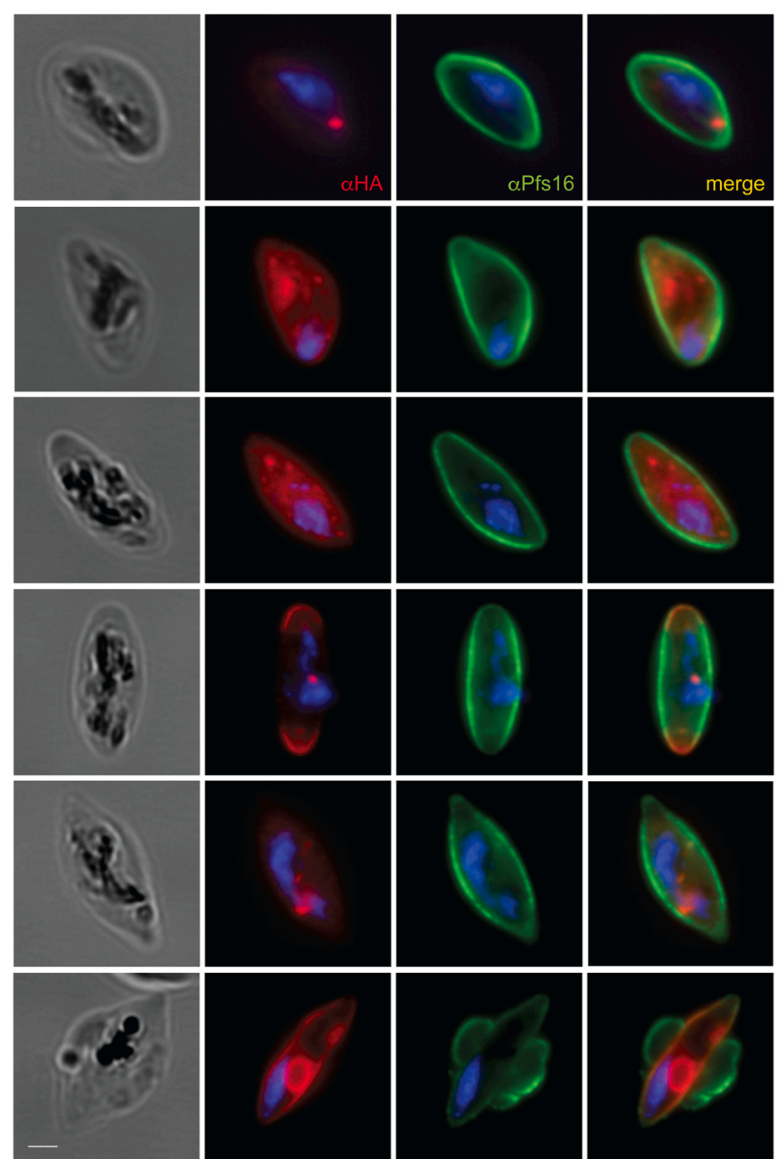

B

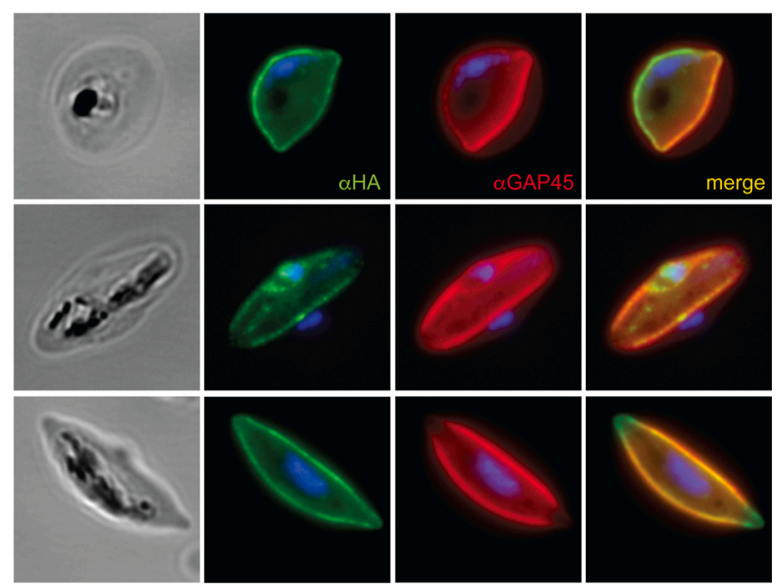

C

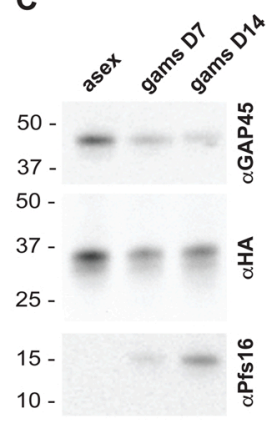

Fig. 7. PfPLSCR is expressed in gametocytes. A) Immunofluorescence analysis of stage III-IV gametocytes reveals different localisation patterns of the triple-HA tagged protein. Anti-Pfs 16 is used as gametocyte specific antibody marking the PVM. Parasite nuclei are stained with DAPI. Scale bar $=1 \mu$ M. B) PfPLSCR-HA colocalises with the IMC marker GAP45, which delineates the parasite periphery. C) Western blot analysis confirms PfPLSCR-HA expression in gametocytes.

gametocyte-specific ATP-binding cassette transporter (gABCG2) in regulating the numbers of gametocytes and accumulation of neutral lipids [59], changes in the lipid profile during sexual parasite development $[3,74]$ and the involvement of host cell derived phospholipids in mediating sexual commitment and gametocytogenesis in $P$. falciparum [75] and liver stage infection [76], respectively, highlight the emerging role of lipids and their regulators throughout the parasite's life cycle and underpin the notion of a putative function for PfPLSCR in the sexual stages of Plasmodium spp. parasites. Various cellular activities have been demonstrated and proposed for hPLSCR1, though the precise biological function of this multifaceted lipid effector and its counterpart in Plasmodium spp. remains to be unravelled.

\section{Author contributions}

S.H. conceived the project, designed, performed and coordinated the experiments as well as analysed and interpreted the data. M.C. optimised recombinant protein expression and purification, as well as D.M. who also provided guidance with the biochemical assays. D.C. assisted in recombinant protein expression and purification. S.J. assisted in preparing $P$. falciparum gametocyte cultures and samples. J.M.G. and J. B. provided scientific and advisory support. S.H. wrote the manuscript. All authors contributed to editing and approving the manuscript.

\section{Author statement}

Silvia Haase: Conceptualization, Methodology, Validation, Formal analysis, Investigation, Data Curation, Writing - Original Draft, Visualisation, Supervision, Project administration. Melanie Condron: Methodology, Validation, Formal analysis, Investigation. David Miller: Methodology, Formal analysis, Investigation. Dounia Cherkaoui: Methodology, Validation, Investigation. Sarah Jordan: Investigation. Jacqueline Gulbis: Resources, Writing - Reviewing and Editing, Funding acquisition. Jake Baum: Resources, Writing - Reviewing and Editing, Funding acquisition.

\section{Declaration of Competing Interest}

The authors report no declarations of interest.

\section{Acknowledgments}

We thank Matt Child for critically reading the manuscript, Moritz Treeck (Francis Crick Institute, UK) and Marcus Lee (Wellcome Sanger Institute, UK) for providing plasmids, as well as Robert Sauerwein (Radboud UMC), Brendan Crabb (Burnet Institute) and Alan Cowman (Walter \& Eliza Hall Institute) for provision of antibodies. We are indebted to Simone Kunzelmann and the Structural Biology STP at the Francis Crick Institute for access to the Jasco FP-8500 Spectrofluorometer and to Marc Morgan at the Imperial College X-ray Crystallography Facility for help with SEC-MALS analysis and crystallisation trials. This research was funded by the Wellcome Trust, Investigator Award to JB $(100993 / Z / 13 / Z)$. For the purpose of open access, the author has applied a CC BY public copyright licence to any Author Accepted 
Manuscript version arising from this submission.

\section{Appendix A. Supplementary data}

Supplementary material related to this article can be found, in the online version, at doi:https://doi.org/10.1016/j.molbiopara.2021. 111374.

\section{References}

[1] H.J. Vial, P. Eldin, A.G.M. Tielens, J.J. van Hellemond, Phospholipids in parasitic protozoa, Mol. Biochem. Parasitol. 126 (2003) 143-154.

[2] E.S. Sherling, C. van Ooij, Host cell remodeling by pathogens: the exomembrane system in Plasmodium-infected erythrocytes, FEMS Microbiol. Rev. 40 (2016) 701-721.

[3] S. Gulati, E.H. Ekland, K.V. Ruggles, R.B. Chan, B. Jayabalasingham, B. Zhou, P. Y. Mantel, M.C. Lee, N. Spottiswoode, O. Coburn-Flynn, et al., Profiling the essential nature of lipid metabolism in asexual blood and gametocyte stages of plasmodium falciparum, Cell Host Microbe 18 (2015) 371-381.

[4] C.W. McNamara, M.C. Lee, C.S. Lim, C.S.H. Lim, J. Roland, O. Simon, B.K. Yeung, A.K. Chatterjee, S.L. McCormack, M.J. Manary, et al., Targeting plasmodium PI(4) K to eliminate malaria, Nature 504 (2013) 248-253.

[5] A. Vaid, R. Ranjan, W.A. Smythe, H.C. Hoppe, Sharma P: PfPI3K, a phosphatidylinositol-3 kinase from Plasmodium falciparum, is exported to the host erythrocyte and is involved in hemoglobin trafficking, Blood 115 (2010) 2500-2507.

[6] Z. Ebrahimzadeh, A. Mukherjee, M.E. Crochetiere, A. Sergerie, S. Amiar, L. A. Thompson, D. Gagnon, D. Gaumond, R.V. Stahelin, J.B. Dacks, D. Richard, A pan-apicomplexan phosphoinositide-binding protein acts in malarial microneme exocytosis, EMBO Rep. (2019) 20.

[7] L. Tawk, J.F. Dubremetz, P. Montcourrier, G. Chicanne, F. Merezegue, V. Richard, B. Payrastre, M. Meissner, H.J. Vial, C. Roy, et al., Phosphatidylinositol 3-monophosphate is involved in toxoplasma apicoplast biogenesis, PLoS Pathog. 7 (2011) e1001286.

[8] L. Tawk, G. Chicanne, J.-F. Dubremetz, V. Richard, B. Payrastre, H.J. Vial, C. Roy, K. Wengelnik, Phosphatidylinositol 3-phosphate, an essential lipid in plasmodium, localizes to the food vacuole membrane and the apicoplast, Eukaryot. Cell 9 (2010) 1519-1530.

[9] H.E. Bullen, Y. Jia, Y. Yamaryo-Botte, H. Bisio, O. Zhang, N.K. Jemelin, J.B. Marq, V. Carruthers, C.Y. Botte, D. Soldati-Favre, Phosphatidic acid-mediated signaling regulates microneme secretion in toxoplasma, Cell Host Microbe 19 (2016) 349-360.

[10] H. Bisio, M. Lunghi, M. Brochet, D. Soldati-Favre, Phosphatidic acid governs natural egress in Toxoplasma gondii via a guanylate cyclase receptor platform, Nat. Microbiol. 4 (2019) 420-428.

[11] A.M. Goldston, R.R. Powell, L.A. Temesvari, Sink or swim: lipid rafts in parasite pathogenesis, Trends Parasitol. 28 (2012) 417-426, 417-426.

[12] R. Dadhich, S. Kapoor, Various facets of pathogenic lipids in infectious diseases: exploring virulent lipid-host interactome and their druggability, J. Membr. Biol. 253 (2020) 399-423.

[13] A. Zachowski, Phospholipids in animal eukaryotic membranes: transverse asymmetry and movement, Biochem. J. 294 (Pt 1) (1993) 1-14.

[14] R.L. Lopez-Marques, L.R. Poulsen, A. Bailly, M. Geisler, T.G. Pomorski, M. G. Palmgren, Structure and mechanism of ATP-dependent phospholipid transporters, Biochim. Biophys. Acta 1850 (2015) 461-475.

[15] K.M. Kodigepalli, K. Bowers, A. Sharp, M. Nanjundan, Roles and regulation of phospholipid scramblases, FEBS Lett. 589 (2015) 3-14.

[16] S. Lourido, S.N. Moreno, The calcium signaling toolkit of the Apicomplexan parasites Toxoplasma gondii and Plasmodium spp, Cell Calcium 57 (2015) 186-193.

[17] B.A. Chua, J.A. Ngo, K. Situ, K. Morizono, Roles of phosphatidylserine exposed on the viral envelope and cell membrane in HIV-1 replication, Cell Commun. Signal 17 (2019) 132.

[18] T. Wiedmer, Q. Zhou, D.Y. Kwoh, P.J. Sims, Identification of three new members of the phospholipid scramblase gene family, Biochim. Biophys. Acta 1467 (2000) 244-253.

[19] F. Basse, J.G. Stout, P.J. Sims, T. Wiedmer, Isolation of an erythrocyte membrane protein that mediates Ca2+-dependent transbilayer movement of phospholipid, J. Biol. Chem. 271 (1996) 17205-17210.

[20] Q. Zhou, J. Zhao, J.G. Stout, R.A. Luhm, T. Wiedmer, P.J. Sims, Molecular cloning of human plasma membrane phospholipid scramblase. A protein mediating transbilayer movement of plasma membrane phospholipids, J. Biol. Chem. 272 (1997) 18240-18244.

[21] Q. Zhou, I. Ben-Efraim, J.L. Bigcas, D. Junqueira, T. Wiedmer, P.J. Sims, Phospholipid scramblase 1 binds to the promoter region of the inositol 1,4,5triphosphate receptor type 1 gene to enhance its expression, J. Biol. Chem. 280 (2005) 35062-35068.

[22] T. Wiedmer, J. Zhao, M. Nanjundan, P.J. Sims, Palmitoylation of phospholipid scramblase 1 controls its distribution between nucleus and plasma membrane, Biochemistry 42 (2003) 1227-1233.

[23] M.H. Chen, I. Ben-Efraim, G. Mitrousis, N. Walker-Kopp, P.J. Sims, G. Cingolani, Phospholipid scramblase 1 contains a nonclassical nuclear localization signal with unique binding site in importin alpha, J. Biol. Chem. 280 (2005) 10599-10606.
[24] Q. Zhou, P.J. Sims, T. Wiedmer, Identity of a conserved motif in phospholipid scramblase that is required for $\mathrm{Ca} 2+$-accelerated transbilayer movement of membrane phospholipids, Biochemistry 37 (1998) 2356-2360.

[25] V.G. Francis, A.M. Mohammed, G.K. Aradhyam, S.N. Gummadi, The single Cterminal helix of human phospholipid scramblase 1 is required for membrane insertion and scrambling activity, FEBS J. 280 (2013) 2855-2869.

[26] J. Zhao, Q. Zhou, T. Wiedmer, P.J. Sims, Level of expression of phospholipid scramblase regulates induced movement of phosphatidylserine to the cell surface, J. Biol. Chem. 273 (1998) 6603-6606.

[27] V.G. Francis, M.A. Majeed, S.N. Gummadi, Recovery of functionally active recombinant human phospholipid scramblase 1 from inclusion bodies using $\mathrm{N}$ lauroyl sarcosine, J. Ind. Microbiol. Biotechnol. 39 (2012) 1041-1048.

[28] S. Rayala, V.G. Francis, U. Sivagnanam, S.N. Gummadi, N-terminal proline-rich domain is required for scrambling activity of human phospholipid scramblases, J. Biol. Chem. 289 (2014) 13206-13218.

[29] S.K. Sahu, G.K. Aradhyam, S.N. Gummadi, Calcium binding studies of peptides of human phospholipid scramblases 1 to 4 suggest that scramblases are new class of calcium binding proteins in the cell, Biochim. Biophys. Acta 1790 (2009) 1274-1281.

[30] S.K. Sahu, A. Gopala Krishna, S.N. Gummadi, Over-expression of recombinant human phospholipid scramblase 1 in E. coli and its purification from inclusion bodies, Biotechnol. Lett. 30 (2008) 2131-2137.

[31] J.D. Brunner, S. Schenck, Preparation of proteoliposomes with purified TMEM16 protein for accurate measures of lipid scramblase activity, Methods Mol. Biol. 1949 (2019) 181-199.

[32] J.D. Brunner, N.K. Lim, S. Schenck, A. Duerst, R. Dutzler, X-ray structure of a calcium-activated TMEM16 lipid scramblase, Nature 516 (2014) 207-212.

[33] M. Tiburcio, A.S.P. Yang, K. Yahata, P. Suarez-Cortes, H. Belda, S. Baumgarten, M. van de Vegte-Bolmer, G.J. van Gemert, Y. van Waardenburg, E.A. Levashina, et al., A novel tool for the generation of conditional knockouts to study gene function across the plasmodium falciparum life cycle, mBio 10 (2019).

[34] M.L. Jones, S. Das, H. Belda, C.R. Collins, M.J. Blackman, M. Treeck, A versatile strategy for rapid conditional genome engineering using loxP sites in a small synthetic intron in Plasmodium falciparum, Sci. Rep. 6 (2016), 21800.

[35] G.W. Ashdown, M. Dimon, M. Fan, F. Sanchez-Roman Teran, K. Witmer, D.C. A. Gaboriau, Z. Armstrong, D.M. Ando, J. Baum, A machine learning approach to define antimalarial drug action from heterogeneous cell-based screens, Sci. Adv. (2020) 6.

[36] J. White, S.K. Dhingra, X. Deng, F. El Mazouni, M.C.S. Lee, G.A. Afanador, A. Lawong, D.R. Tomchick, C.L. Ng, J. Bath, et al., Identification and mechanistic understanding of dihydroorotate dehydrogenase point mutations in Plasmodium falciparum that confer in vitro resistance to the clinical candidate DSM265, ACS Infect. Dis. 5 (2019) 90-101.

[37] K. Labun, T.G. Montague, J.A. Gagnon, S.B. Thyme, E. Valen, CHOPCHOP v2: a web tool for the next generation of CRISPR genome engineering, Nucleic Acids Res. 44 (2016) W272-276.

[38] W. Trager, J.B. Jensen, Human malaria parasites in continuous culture, Science 193 (1976) 673-675.

[39] A.J. Perrin, C.R. Collins, M.R.G. Russell, L.M. Collinson, D.A. Baker, M. J. Blackman, The actinomyosin motor drives malaria parasite red blood cell invasion but not egress, MBio (2018) 9.

[40] J. Birnbaum, S. Flemming, N. Reichard, A.B. Soares, P. Mesen-Ramirez, E. Jonscher, B. Bergmann, T. Spielmann, A genetic system to study Plasmodium falciparum protein function, Nat. Methods 14 (2017) 450-456.

[41] W. Wong, C.T. Skau, D.S. Marapana, E. Hanssen, N.L. Taylor, D.T. Riglar, E. S. Zuccala, F. Angrisano, H. Lewis, B. Catimel, et al., Minimal requirements for actin filament disassembly revealed by structural analysis of malaria parasite actindepolymerizing factor 1, Proc. Natl. Acad. Sci. U. S. A. 108 (2011) 9869-9874.

[42] F. Angrisano, D.T. Riglar, A. Sturm, J.C. Volz, M.J. Delves, E.S. Zuccala, L. Turnbull, C. Dekiwadia, M.A. Olshina, D.S. Marapana, et al., Spatial localisation of actin filaments across developmental stages of the malaria parasite, PLoS One 7 (2012), e32188.

[43] D.W. Wilson, F.J. Fowkes, P.R. Gilson, S.R. Elliott, L. Tavul, P. Michon, E. Dabod, P. M. Siba, I. Mueller, B.S. Crabb, J.G. Beeson, Quantifying the importance of MSP119 as a target of growth-inhibitory and protective antibodies against Plasmodium falciparum in humans, PLoS One 6 (2011), e27705.

[44] D.S. Marapana, D.W. Wilson, E.S. Zuccala, C.D. Dekiwadia, J.G. Beeson, S. A. Ralph, J. Baum, Malaria parasite signal peptide peptidase is an ER-resident protease required for growth but not for invasion, Traffic 13 (2012) 1457-1465.

[45] J. Baum, D. Richard, J. Healer, M. Rug, Z. Krnajski, T.W. Gilberger, J.L. Green, A. A. Holder, A.F. Cowman, A conserved molecular motor drives cell invasion and gliding motility across malaria life cycle stages and other apicomplexan parasites, J. Biol. Chem. 281 (2006) 5197-5208.

[46] J. Schindelin, I. Arganda-Carreras, E. Frise, V. Kaynig, M. Longair, T. Pietzsch, S. Preibisch, C. Rueden, S. Saalfeld, B. Schmid, et al., Fiji: an open-source platform for biological-image analysis, Nat. Methods 9 (2012) 676-682.

[47] F. de Chaumont, S. Dallongeville, N. Chenouard, N. Herve, S. Pop, T. Provoost, V. Meas-Yedid, P. Pankajakshan, T. Lecomte, Y. Le Montagner, et al., Icy: an open bioimage informatics platform for extended reproducible research, Nat. Methods 9 (2012) 690-696.

[48] P. Stothard, The sequence manipulation suite: JavaScript programs for analyzing and formatting protein and DNA sequences, Biotechniques 28 (1102) (2000) 1104.

[49] J. Ren, L. Wen, X. Gao, C. Jin, Y. Xue, X. Yao, CSS-Palm 2.0: an updated software for palmitoylation sites prediction, Protein Eng. Des. Sel. 21 (2008) 639-644. 
[50] S. Kosugi, M. Hasebe, M. Tomita, H. Yanagawa, Systematic identification of cell cycle-dependent yeast nucleocytoplasmic shuttling proteins by prediction of composite motifs, Proc. Natl. Acad. Sci. U. S. A. 106 (2009) 10171-10176.

[51] K.D. Tsirigos, C. Peters, N. Shu, L. Kall, A. Elofsson, The TOPCONS web server for consensus prediction of membrane protein topology and signal peptides, Nucleic Acids Res. 43 (2015) W401-407.

[52] S.K. Sahu, S.N. Gummadi, N. Manoj, G.K. Aradhyam, Phospholipid scramblases: an overview, Arch. Biochem. Biophys. 462 (2007) 103-114.

[53] R.B. Moore, M. Oborník, J. Janouškovec, T. Chrudimský, M. Vancová, D.H. Green, S.W. Wright, N.W. Davies, C.J.S. Bolch, K. Heimann, et al., A photosynthetic alveolate closely related to apicomplexan parasites, Nature 451 (2008) 959-963.

[54] J. Janouskovec, A. Horak, K.L. Barott, F.L. Rohwer, P.J. Keeling, Global analysis of plastid diversity reveals apicomplexan-related lineages in coral reefs, Curr. Biol. 22 (2012) R518-519.

[55] M. Obornik, J. Lukes, Cell biology of chromerids: autotrophic relatives to apicomplexan parasites, Int. Rev. Cell Mol. Biol. 306 (2013) 333-369.

[56] J.G. Stout, Q. Zhou, T. Wiedmer, P.J. Sims, Change in conformation of plasma membrane phospholipid scramblase induced by occupancy of its Ca2+ binding site, Biochemistry 37 (1998) 14860-14866.

[57] L. Sanchez-Magraner, I.M. Posada, N. Andraka, F.X. Contreras, A.R. Viguera, D. M. Guerin, J.L. Arrondo, H.L. Monaco, Goni FM, The C-terminal transmembrane domain of human phospholipid scramblase 1 is essential for the protein flip-flop activity and $\mathrm{Ca}(2)(+)$-binding, J. Membr. Biol. 247 (2014) 155-165.

[58] T. Venken, A.S. Schillinger, E. Fuglebakk, Reuter N, Interactions stabilizing the Cterminal helix of human phospholipid scramblase 1 in lipid bilayers: a computational study, Biochim. Biophys. Acta Biomembr. 1859 (2017) 1200-1210.

[59] P.N. Tran, S.H. Brown, T.W. Mitchell, K. Matuschewski, P.J. McMillan, K. Kirk, M. W. Dixon, A.G. Maier, A female gametocyte-specific ABC transporter plays a role in lipid metabolism in the malaria parasite, Nat. Commun. 5 (2014) 4773.

[60] M.K. Dearnley, J.A. Yeoman, E. Hanssen, S. Kenny, L. Turnbull, C.B. Whitchurch, L. Tilley, M.W. Dixon, Origin, composition, organization and function of the inner membrane complex of Plasmodium falciparum gametocytes, J. Cell. Sci. 125 (2012) 2053-2063.

[61] N. Arashiki, M. Saito, I. Koshino, K. Kamata, J. Hale, N. Mohandas, S. Manno, Y. Takakuwa, An unrecognized function of cholesterol: regulating the mechanism controlling membrane phospholipid asymmetry, Biochemistry 55 (2016) 3504-3513.

[62] V. Kalienkova, V. Clerico Mosina, L. Bryner, G.T. Oostergetel, R. Dutzler, C. Paulino, Stepwise activation mechanism of the scramblase nhTMEM16 revealed by cryo-EM, Elife 8 (2019).

[63] B. Ploier, A.K. Menon, A fluorescence-based assay of phospholipid scramblase activity, J. Vis. Exp. (2016).

[64] M. Malvezzi, M. Chalat, R. Janjusevic, A. Picollo, H. Terashima, A.K. Menon, Accardi A, Ca2+-dependent phospholipid scrambling by a reconstituted TMEM16 ion channel, Nat. Commun. 4 (2013), 2367.

[65] K. Pandey, P.E. Ferreira, T. Ishikawa, T. Nagai, O. Kaneko, K. Yahata, Ca(2+) monitoring in Plasmodium falciparum using the yellow cameleon-Nano biosensor, Sci. Rep. 6 (2016), 23454.
[66] S.K. Palanirajan, U. Sivagnanam, S. Murugan, S.N. Gummadi, In vitro reconstitution and biochemical characterization of human phospholipid scramblase 3: phospholipid specificity and metal ion binding studies, Biol. Chem. 399 (2018) 361-374.

[67] S.C. Frasch, P.M. Henson, J.M. Kailey, D.A. Richter, M.S. Janes, V.A. Fadok, Bratton DL, Regulation of phospholipid scramblase activity during apoptosis and cell activation by protein kinase Cdelta, J. Biol. Chem. 275 (2000) 23065-23073.

[68] M. Treeck, J.L. Sanders, J.E. Elias, J.C. Boothroyd, The phosphoproteomes of Plasmodium falciparum and Toxoplasma gondii reveal unusual adaptations within and beyond the parasites\&apos; boundaries, Cell Host Microbe 10 (2011) 410-419.

[69] B.A. Merrick, S. Dhungana, J.G. Williams, J.J. Aloor, S. Peddada, K.B. Tomer, M. B. Fessler, Proteomic profiling of S-acylated macrophage proteins identifies a role for palmitoylation in mitochondrial targeting of phospholipid scramblase 3, Mol. Cell Proteomics 10 (M110) (2011), 006007.

[70] K. Lott, A. Bhardwaj, P.J. Sims, G. Cingolani, A minimal nuclear localization signal (NLS) in human phospholipid scramblase 4 that binds only the minor NLS-binding site of importin alpha1, J. Biol. Chem. 286 (2011) 28160-28169.

[71] I. Ben-Efraim, Q. Zhou, T. Wiedmer, L. Gerace, P.J. Sims, Phospholipid scramblase 1 is imported into the nucleus by a receptor-mediated pathway and interacts with DNA, Biochemistry 43 (2004) 3518-3526.

[72] M.L. Jones, M.O. Collins, D. Goulding, J.S. Choudhary, J.C. Rayner, Analysis of protein palmitoylation reveals a pervasive role in Plasmodium development and pathogenesis, Cell Host Microbe 12 (2012) 246-258.

[73] M. Zhang, C. Wang, T.D. Otto, J. Oberstaller, X. Liao, S.R. Adapa, K. Udenze, I. F. Bronner, D. Casandra, M. Mayho, et al., Uncovering the essential genes of the human malaria parasite Plasmodium falciparum by saturation mutagenesis, Science 360 (2018)

[74] P.N. Tran, S.H. Brown, M. Rug, M.C. Ridgway, T.W. Mitchell, A.G. Maier, Changes in lipid composition during sexual development of the malaria parasite Plasmodium falciparum, Malar. J. 15 (2016) 73.

[75] N.M.B. Brancucci, J.P. Gerdt, C. Wang, M. De Niz, N. Philip, S.R. Adapa, M. Zhang, E. Hitz, I. Niederwieser, S.D. Boltryk, et al., Lysophosphatidylcholine regulates sexual stage differentiation in the human malaria parasite plasmodium falciparum, Cell 171 (1532-1544) (2017) e1515.

[76] M.A. Itoe, J.L. Sampaio, G.G. Cabal, E. Real, V. Zuzarte-Luis, S. March, S.N. Bhatia, F. Frischknecht, C. Thiele, A. Shevchenko, M.M. Mota, Host cell phosphatidylcholine is a key mediator of malaria parasite survival during liver stage infection, Cell Host Microbe 16 (2014) 778-786.

[77] F. Madeira, Y.M. Park, J. Lee, N. Buso, T. Gur, N. Madhusoodanan, P. Basutkar, A. R.N. Tivey, S.C. Potter, R.D. Finn, R. Lopez, The EMBL-EBI search and sequence analysis tools APIs in 2019, Nucleic Acids Res. 47 (2019) W636-W641.

[78] M.A. Larkin, G. Blackshields, N.P. Brown, R. Chenna, P.A. McGettigan, H. McWilliam, F. Valentin, I.M. Wallace, A. Wilm, R. Lopez, et al., Clustal W and clustal X version 2.0, Bioinformatics 23 (2007) 2947-2948.

[79] I. Letunic, P. Bork, Interactive Tree of Life (iTOL) v4: recent updates and new developments, Nucleic Acids Res. 47 (2019) W256-W259. 


\section{University Library}

\section{- M M N E R VA A gateway to Melbourne's research publications}

Minerva Access is the Institutional Repository of The University of Melbourne

Author/s:

Haase, S;Condron, M;Miller, D;Cherkaoui, D;Jordan, S;Gulbis, JM;Baum, J

Title:

Identification and characterisation of a phospholipid scramblase in the malaria parasite Plasmodium falciparum

Date:

2021-05-18

Citation:

Haase, S., Condron, M., Miller, D., Cherkaoui, D., Jordan, S., Gulbis, J. M. \& Baum, J. (2021). Identification and characterisation of a phospholipid scramblase in the malaria parasite Plasmodium falciparum. MOLECULAR AND BIOCHEMICAL PARASITOLOGY, 243, https://doi.org/10.1016/j.molbiopara.2021.111374.

Persistent Link:

http://hdl.handle.net/11343/281753

License:

CC BY 